\title{
Safety, tolerability and antiviral activity of the antisense oligonucleotide bepirovirsen in patients with chronic hepatitis $B$ : a phase 2 randomized controlled trial
}

\author{
Man-Fung Yuen ${ }^{1 凶}$, Jeong Heo (10) ${ }^{2}$, Jeong-Won Jang ${ }^{3}$, Jung-Hwan Yoon ${ }^{4}$, Young-Oh Kweon ${ }^{5}$, \\ Sung-Jae Park ${ }^{6}$, Yvonne Tami ${ }^{7}$, Shihyun You ${ }^{8}$, Phillip Yates ${ }^{9}$, Yu Tao ${ }^{8}$, Jennifer Cremer ${ }^{10}$, \\ Fiona Campbell ${ }^{9}$, Robert Elston ${ }^{9}$, Dickens Theodore ${ }^{10}$, Melanie Paff $\mathbb{D}^{8}{ }^{8}$, C. Frank Bennett $^{7}$ and \\ T. Jesse Kwoh ${ }^{7}$
}

Chronic infection with hepatitis B virus (HBV) leads to an increased risk of death from cirrhosis and hepatocellular carcinoma. Functional cure rates are low with current treatment options (nucleos(t)ide analogs (NAs) and pegylated interferons). Bepirovirsen is an antisense oligonucleotide targeting all HBV messenger RNAs; in cell culture and animal models, bepirovirsen leads to reductions in HBV-derived RNAs, HBV DNA and viral proteins. This phase 2 double-blinded, randomized, placebo-controlled trial is the first evaluation of the safety and activity of an antisense oligonucleotide targeting HBV RNA in both treatment-naïve and virally suppressed individuals with chronic HBV infection. The primary objective was to assess the safety and tolerability of bepirovirsen in individuals with chronic hepatitis B (CHB) (NCT02981602). The secondary objective was to assess antiviral activity, including the change from baseline to day 29 in serum hepatitis B surface antigen (HBsAg) concentration. Participants with CHB infection $\geq 6$ months and serum $\mathrm{HBsAg} \geq 50 \mathrm{IU} \mathrm{ml}^{-1}$ were enrolled from seven centers across Hong Kong and the Republic of Korea and randomized (3:1 within each dose cohort) to receive bepirovirsen or placebo via subcutaneous injection twice weekly during weeks 1 and 2 (days 1, 4, 8 and 11) and once weekly during weeks 3 and 4 (days 15 and 22). Participants were then followed for 26 weeks. Twenty-four participants were treatment-naïve and seven were receiving stable NA therapy. Treatment-emergent adverse events were mostly mild/moderate (most commonly injection site reactions). Eleven $(61.1 \%)$ and three $(50.0 \%)$ treatment-naïve participants experienced one or more treatment-emergent adverse event in the bepirovirsen and placebo groups, respectively. In participants receiving NA therapy, the corresponding numbers were three $(60.0 \%)$ and one $(50.0 \%)$. Transient, self-resolving alanine aminotransferase flares ( $\geq 2 \times$ upper limit of normal) were observed in eight treatment-naïve participants and three participants on stable NA regimens in the bepirovirsen treatment arms. HBsAg reductions were observed and were significant versus placebo for treatment-naïve participants receiving bepirovirsen $300 \mathrm{mg}(P=0.001)$, but not for the bepirovirsen $150 \mathrm{mg}$ group $(P=0.245)$ or participants receiving stable $N A$ therapy $(P=0.762)$. Two participants in each of the $300 \mathrm{mg}$ dose groups achieved HBsAg levels below the lower limit of quantitation by day $29(n=3)$ or day $36(n=1)$. Bepirovirsen had a favorable safety profile. These preliminary observations warrant further investigation of the safety and activity of bepirovirsen in a larger CHB patient population.

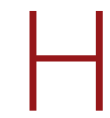
BV infection can be chronic, especially if it occurs before 5 years of age, and chronicity results in an increased risk of death from cirrhosis and hepatocellular carcinoma in the long term ${ }^{1}$. In 2019, it was estimated that 296 million people were living with chronic HBV infection, resulting in $\sim 820,000$ deaths per year globally in 2019, mostly from cirrhosis and hepatocellular carcinoma (https://www.who.int/news-room/fact-sheets/detail/ hepatitis-b $)^{2}$. The World Health Organization's global target is to reduce new viral hepatitis infections by $90 \%$ by 2030 (https://www. who.int/news-room/fact-sheets/detail/hepatitis-b). The treatment goal for patients with $\mathrm{CHB}$ is to obtain functional cure, namely, sustained loss of detectable serum HBsAg with undetectable HBV $\mathrm{DNA}^{3}$. Functional cure is associated with improved clinical outcomes and allows patients to be off-therapy with a minimal risk of relapse $e^{4-6}$. HBsAg plays a major role in maintaining chronic $\mathrm{HBV}$ infection by impairing the immune response against $\mathrm{HBV}^{7,8}$. Reducing HBsAg expression may permit reconstitution of an immune response against $\mathrm{HBV}^{7}$.

First-line therapy for $\mathrm{CHB}$ is $\mathrm{NAs}^{1,9}$. Current NAs suppress serum HBV DNA levels and improve hepatic fibrosis and cirrhosis $^{1,4}$, but have minimal effect on HBsAg levels ${ }^{10,11}$. Even with life-long therapy, HBsAg seroclearance is uncommon ${ }^{9}$ and there is

'Department of Medicine and State Key Laboratory of Liver Research, Queen Mary Hospital, The University of Hong Kong, Hong Kong, China. ${ }^{2}$ College of Medicine, Pusan National University and Biomedical Research Institute, Pusan National University Hospital, Busan, Republic of Korea. ${ }^{3}$ Seoul St. Mary's Hospital, The Catholic University of Korea, Seoul, Republic of Korea. ${ }^{4}$ Seoul National University Hospital, Seoul, Republic of Korea. ${ }^{5}$ Kyungpook National University Hospital, Daegu, Republic of Korea. ${ }^{6}$ Inje University Busan Paik Hospital, Busan, Republic of Korea. ${ }^{7}$ lonis Pharmaceuticals Inc, Carlsbad, CA, USA. ${ }^{8}$ GlaxoSmithKline, Collegeville, PA, USA. ${ }^{9} \mathrm{GlaxoSmithKline,} \mathrm{Stevenage,} \mathrm{UK.}{ }^{10} \mathrm{GlaxoS}$ ithKline, Research Triangle Park, NC, USA.

凶e-mail: mfyuen@hkucc.hku.hk 
a high relapse rate after NA withdrawal due to the persistence in hepatocytes of covalently closed circular HBV DNA, which is the transcription template enabling the resumption of HBV DNA replication after NA treatment is ended ${ }^{10,12}$. Pegylated interferons are also approved for CHB treatment for up to 48 weeks ${ }^{5,9}$. However, the tolerability profile of approved pegylated interferons results in many patients being ineligible or unwilling to receive treatment ${ }^{9}$. Treatment-induced functional cure rates are low with current treatment options?.

There is a significant unmet need for new CHB therapies that achieve functional cure when administered for a finite duration, enabling patients to control their infection, freeing them from life-long therapy and reducing their risk of hepatocellular carcinoma. Bepirovirsen (previously ISIS 505358; GSK3228836) is an antisense oligonucleotide (ASO) with a $2^{\prime}$-O-methoxyethyl $\left(2^{\prime}\right.$-MOE) gapmer design (full sequence is publicly available) ${ }^{13}$. The first five and final five nucleotides are of MOE-modified ribonucleotides; the central ten nucleotides are oligodeoxynucleotides. The drug is uniformly modified with phosphorothioate linkages. The bepirovirsen binding site (GCACTTCGCTTCACCTCTGC) is present in all HBV mRNA and pregenomic RNA; as such, bepirovirsen would be expected to reduce levels of all HBV mRNAs including pregenomic RNA. Specific ASOs bind to complementary HBV RNA transcripts forming a hybrid ASO/RNA complex, which recruits endogenous RNase $\mathrm{H}$, cleaving the HBV RNA and leading to degradation of the transcript. This results in a reduction in HBV-derived RNAs, HBV DNA and viral proteins (including HBsAg) in cell culture and in animal models ${ }^{14}$.

This study examined the safety, tolerability and antiviral activity of multiple doses of bepirovirsen over 4 weeks in patients with $\mathrm{CHB}$.

\section{Results}

Study participants and dosing. The ISIS 505358-CS3 study (GSK study 205695; ClinicalTrials.gov: NCT02981602) was a phase 2, double-blinded, placebo-controlled, dose-escalation trial of bepirovirsen in 31 patients with $\mathrm{CHB}$ who were either treatment-naïve (cohorts $1-3, n=24$ ) or receiving stable NA therapy (cohort 4 (on-NA), $n=7$ ) (Supplementary Fig. 1). The study protocol can be accessed at https://www.gsk-studyregister.com/ en/trial-details/?id=205695. Treatment-naïve patients were randomized to placebo $(n=6)$, bepirovirsen $150 \mathrm{mg}(n=6)$ or $300 \mathrm{mg}$ $(n=12)$ and on-NA patients to placebo $(n=2)$ or bepirovirsen $300 \mathrm{mg}(n=5)$ (Supplementary Fig. 2). Six doses of bepirovirsen or placebo were administered via subcutaneous injection during the 4-week treatment period: twice weekly during weeks 1 and 2 (days $1,4,8$ and 11) and once weekly during weeks 3 and 4 (days 15 and 22). Patients were then followed for 26 weeks.

One on-NA patient withdrew from the study on day 8 , after two doses of bepirovirsen $300 \mathrm{mg}$, due to transient mild fevers after each dose that were considered treatment-related; all other patients completed the treatment and follow-up periods. Demographics and baseline characteristics were similar between treatment arms (Table 1).

Safety and tolerability. The most common treatment-emergent adverse events (TEAEs) were local injection site reactions (Table 2). Injection site reactions were reported in zero, three (50\%) and three (25\%) treatment-naïve patients in the placebo, bepirovirsen $150 \mathrm{mg}$ and bepirovirsen $300 \mathrm{mg}$ arms, respectively, and in zero and two (40\%) on-NA patients in the placebo and bepirovirsen $300 \mathrm{mg}$ arms, respectively. Pyrexia was commonly reported in treatment-naïve patients (one patient (16.7\%) each in the placebo and bepirovirsen $150 \mathrm{mg}$ arms; three patients (25\%) in the bepirovirsen $300 \mathrm{mg}$ arm).

TEAEs were mostly mild (division of acquired immune deficiency syndrome (DAIDS) grade 1: 52 of 69 events); the remainder were moderate (DAIDS grade 2: 16 of 69 events), except for one treatment-naïve patient in the bepirovirsen $300 \mathrm{mg}$ treatment group who experienced a serious adverse event (DAIDS grade 4) of alanine aminotransferase (ALT) increase (described in the 'ALT increase' section).

Treatment-related TEAEs were reported in four $(66.7 \%)$, six (50.0\%) and one (16.7\%) treatment-naïve patients in the bepirovirsen $150 \mathrm{mg}$, bepirovirsen $300 \mathrm{mg}$ and placebo groups, respectively. The most common treatment-related TEAEs in treatment-naïve patients were injection site pruritus (bepirovirsen $150 \mathrm{mg}, n=2$ (33\%); bepirovirsen $300 \mathrm{mg}, n=1(8.3 \%)$ ), injection site erythema (bepirovirsen $150 \mathrm{mg}, n=0$ (0\%); bepirovirsen $300 \mathrm{mg}, n=3(25 \%)$ ) and nausea (bepirovirsen $150 \mathrm{mg}, n=1$ (16.7\%); bepirovirsen $300 \mathrm{mg}, n=2(16.7 \%))$. Injection site swelling, ALT increase and myalgia were each considered treatment-related in two patients $(16.7 \%)$ in the bepirovirsen $300 \mathrm{mg}$ arm. In on-NA patients, injection site bruising, injection site swelling, injection site erythema and pyrexia were each reported in one patient $(20.0 \%)$ in the bepirovirsen $300 \mathrm{mg}$ arm.

An increase in C-reactive protein (CRP) levels following the first dose of bepirovirsen was observed in patients with $\mathrm{CHB}$. In most patients, levels increased on day 2 with peak levels observed pre-dose on day 4 (see example patient in Supplementary Fig. 3a). Levels were substantially recovered by day 8 , suggesting the day 4 dose did not lead to further CRP increases. There were generally no CRP spikes at later time points, and no symptoms were consistently associated with CRP elevations. CRP elevations were dose-related in patients and were consistent with those observed in healthy volunteers (Supplementary Fig. 3b and unpublished data).

Aside from ALT, aspartate aminotransferase (AST; described below) and CRP effects, there were no clinically significant changes in laboratory tests related to bepirovirsen treatment. Transient prolongations in activated partial thromboplastin time were observed 3-5 h after administration of both bepirovirsen doses on day 1 and day 22. The magnitudes of these prolongations were not clinically relevant, with a maximum observed value of $43.9 \mathrm{~s}(\sim 1.21 \times$ upper limit of normal (ULN); ULN =36.5 s) in two participants in the bepirovirsen $300 \mathrm{mg}$ treatment group. There were no bleeding or bruising events associated with the elevations and little or no coincidental prolongation of prothrombin time.

There were no observations of complement activation related to bepirovirsen dosing and no clinically significant findings in other safety assessments (for example, electrocardiogram, physical examination, concomitant medication usage).

Efficacy. Change from baseline in HBsAg. A dose-dependent reduction from baseline in HBsAg was observed at day 29 (7 d after the last dose) in the bepirovirsen treatment arms. For treatment-naïve patients, the mean (s.d.) HBsAg reduction from baseline to day 29 was $0.50(0.57) \log _{10} \mathrm{IU} \mathrm{ml} \mathrm{m}^{-1}(P=0.245$ versus placebo $)$ and 1.56 (1.38) $\log _{10} \mathrm{IU} \mathrm{ml}^{-1}(P=0.001$ versus placebo) in the bepirovirsen $150 \mathrm{mg}$ and $300 \mathrm{mg}$ arms, respectively (Table 3 ). For on-NA patients, the mean (s.d.) HBsAg reduction to day 29 was 1.99 (1.80) $\log _{10} \mathrm{IU} \mathrm{ml}^{-1}$ (Table 3 ). By contrast, no placebo-treated patient had a reduction in HBsAg of $>0.07 \log _{10} \mathrm{IU} \mathrm{ml} \mathrm{ml}^{-1}$ by day 29. Four patients achieved transient HBsAg loss, defined as HBsAg level below the lower limit of quantitation (LLOQ: $\left.0.05 \mathrm{IU} \mathrm{ml}^{-1}\right)$, by day $29(n=3)$ or by day $36(n=1)$.

Six of 12 treatment-naïve patients receiving bepirovirsen $300 \mathrm{mg}$ had HBsAg reduction $\geq 1.0 \log _{10} \mathrm{IU} \mathrm{ml^{-1 }}$ at day 29 (an additional patient had $0.98 \log _{10}$-transformed reduction) (Fig. 1a). Three of these six patients experienced a reduction of $\geq 3.0 \log _{10}$ and two achieved HBsAg loss. HBsAg loss was transient in one patient (Fig. 2 and Supplementary Fig. 4c), from day 29 to day 57, with measurable levels $\left(0.14 \mathrm{IU} \mathrm{ml}^{-1}\right)$ detected on day 85 , but was more prolonged in the second patient (from day 23 maintained to day 
Table 1 | Demographics and baseline characteristics in patients with CHB (safety population)

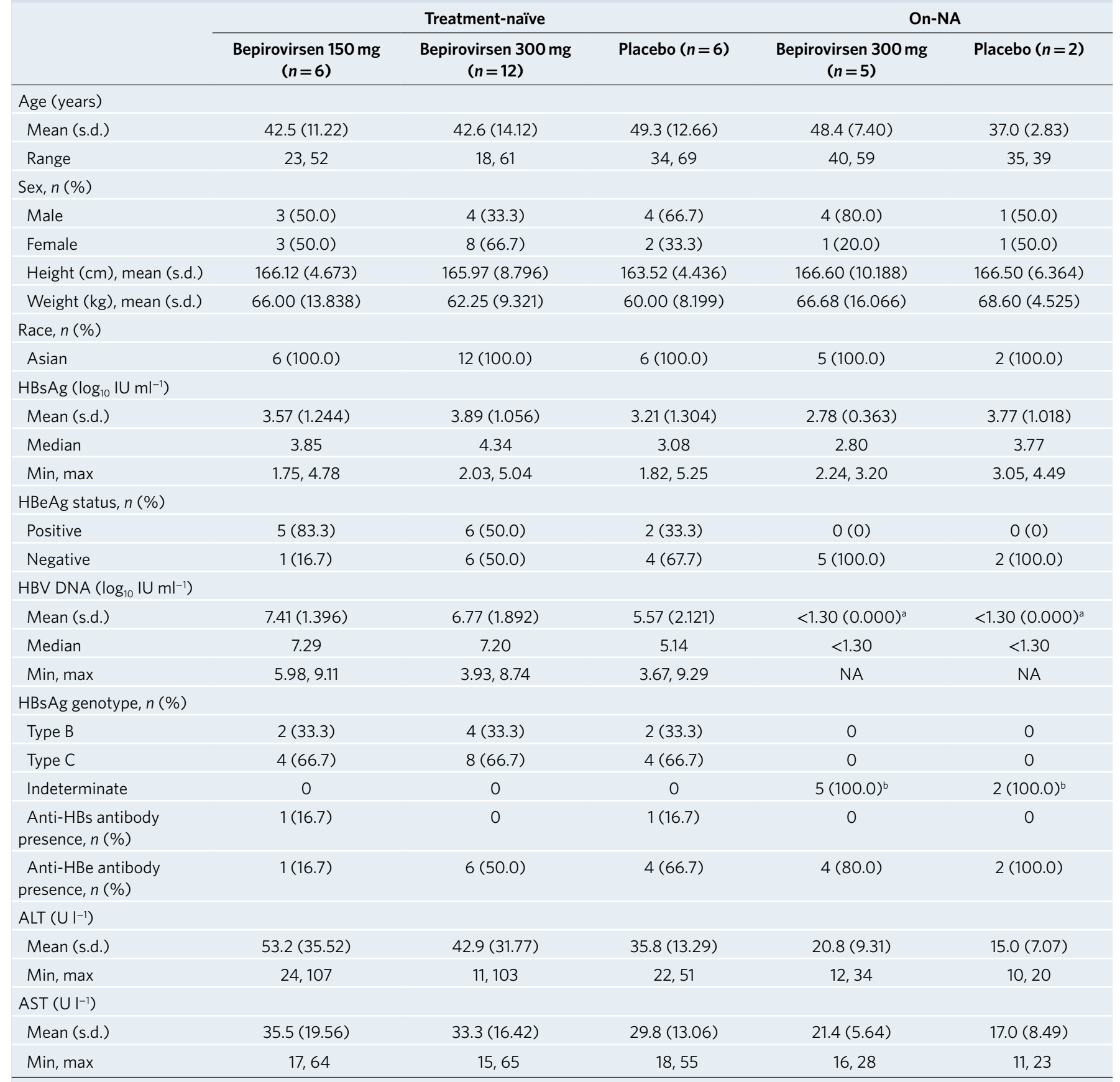

aBecause all participants in cohort 4 were stably maintained on NA, they had undetectable HBV DNA levels at baseline. This table presents log ${ }_{10}$ values of either $<20 \mathrm{IU}^{\mathrm{ml}}{ }^{-1}$ or 'target not detected'. 'Historical data not available and HBV DNA levels were below the LLOQ to establish genotyping. Anti-HBe antibody, antibody to HBV e antigen; anti-HBs, antibody to HBV surface antigen.

126, with measurable levels on day $\left.140\left(0.14 \mathrm{IU} \mathrm{ml}^{-1}\right)\right)($ Fig. 2 and Supplementary Fig. 4).

Three of four on-NA patients completing bepirovirsen $300 \mathrm{mg}$ dosing had a HBsAg reduction $\geq 3.0 \log _{10}$ at day 29 ; of these three patients, one reached HBsAg levels below LLOQ on day 29 and another on day 36, whereas the third patient did not reach HBsAg levels below LLOQ. Similar to the treatment-naïve patients, one patient had transient HBsAg loss (Fig. 2), from day 23 to day 57, with measurable levels detected on day $85\left(0.14 \mathrm{IU} \mathrm{ml}^{-1}\right)$, but a second patient experienced a more prolonged HBsAg loss from day 36 to day 113, inclusive, with measurable levels detected on day 211 (0.12 IU ml-1; maintaining a $3.59 \log _{10} \mathrm{IU} \mathrm{ml}^{-1}$ lower HBsAg level than baseline by the end of the study; Fig. 2 and Supplementary
Fig. 4). The fourth patient had no decrease in HBsAg. Individual HBsAg levels over time by baseline HBsAg concentration are shown in Supplementary Fig. 5.

Reductions in HBsAg were detected in both hepatitis B e-antigen (HBeAg)-positive and HBeAg-negative patients, providing confidence that the target sequence for bepirovirsen is present even when HBsAg may be derived from integrated genomes ${ }^{15}$. Baseline HBsAg levels were generally lower in HBeAg-negative patients than in $\mathrm{HBeAg}$-positive patients, and the $\log _{10}$-transformed reduction in HBsAg from baseline to day 29 was generally of a greater magnitude in $\mathrm{HBeAg}$-negative patients compared with $\mathrm{HBeAg}$-positive patients treated with bepirovirsen $300 \mathrm{mg}$ (Fig. 1a). All four patients who reached undetectable HBsAg levels were HBeAg negative. 
Table 2 | Summary of TEAEs by system organ class and preferred term in patients with CHB (safety population)

\begin{tabular}{|c|c|c|c|c|c|c|}
\hline \multirow{2}{*}{$\begin{array}{l}\text { TEAE, } n(\%) \text { system organ class preferred } \\
\text { term }\end{array}$} & \multicolumn{4}{|c|}{ Treatment-naïve } & \multicolumn{2}{|c|}{ On-NA } \\
\hline & $\begin{array}{l}\text { Bepirovirsen } \\
150 \mathrm{mg}(n=6)\end{array}$ & $\begin{array}{l}\text { Bepirovirsen } \\
300 \mathrm{mg}(n=12)\end{array}$ & $\begin{array}{c}\text { Total } \\
(n=18)\end{array}$ & $\begin{array}{l}\text { Placebo } \\
(n=6)\end{array}$ & $\begin{array}{l}\text { Bepirovirsen } \\
300 \mathrm{mg}(n=5)\end{array}$ & $\begin{array}{l}\text { Placebo } \\
(n=2)\end{array}$ \\
\hline Patients with $\geq 1 \mathrm{TEAE}$ & $5(83.3)$ & $6(50.0)$ & $11(61.1)$ & $3(50.0)$ & $3(60.0)$ & $1(50.0)$ \\
\hline $\begin{array}{l}\text { General disorders and administration site } \\
\text { conditions }\end{array}$ & $4(66.7)$ & $5(41.7)$ & $9(50.0)$ & $2(33.3)$ & $3(60.0)$ & 0 \\
\hline Chest discomfort & 0 & $1(8.3)$ & $1(5.6)$ & 0 & 0 & 0 \\
\hline Injection site bruising & $1(16.7)$ & 0 & $1(5.6)$ & 0 & $1(20.0)$ & 0 \\
\hline Injection site erythema & 0 & $3(25.0)$ & $3(16.7)$ & 0 & $1(20.0)$ & 0 \\
\hline Injection site pain & $1(16.7)$ & $1(8.3)$ & $2(11.1)$ & 0 & 0 & 0 \\
\hline Injection site pruritus & $2(33.3)$ & $1(8.3)$ & $3(16.7)$ & 0 & 0 & 0 \\
\hline Injection site rash & $1(16.7)$ & 0 & $1(5.6)$ & 0 & 0 & 0 \\
\hline Abdominal discomfort & $1(16.7)$ & 0 & $1(5.6)$ & 0 & 0 & 0 \\
\hline Abdominal pain upper & 0 & $1(8.3)$ & $1(5.6)$ & 0 & 0 & 0 \\
\hline Gastritis & 0 & 0 & 0 & $1(16.7)$ & 0 & 0 \\
\hline Mouth swelling & $1(16.7)$ & 0 & $1(5.6)$ & 0 & 0 & 0 \\
\hline Nausea & $1(16.7)$ & $2(16.7)$ & $3(16.7)$ & $1(16.7)$ & 0 & 0 \\
\hline Investigations & 0 & $3(25.0)$ & $3(16.7)$ & 0 & 0 & 0 \\
\hline ALT increased & 0 & $2(16.7)$ & $2(11.1)$ & 0 & 0 & 0 \\
\hline CRP increased & 0 & $1(8.3)$ & $1(5.6)$ & 0 & 0 & 0 \\
\hline Blood and lymphatic system disorders & $1(16.7)$ & $1(8.3)$ & $2(11.1)$ & 0 & 0 & 0 \\
\hline Nervous system disorders & 0 & $2(16.7)$ & $2(11.1)$ & $1(16.7)$ & 0 & $1(50.0)$ \\
\hline Headache & 0 & $2(16.7)$ & $2(11.1)$ & $1(16.7)$ & 0 & $1(50.0)$ \\
\hline Skin and subcutaneous tissue disorders & 0 & $2(16.7)$ & $2(11.1)$ & $1(16.7)$ & 0 & 0 \\
\hline Post inflammatory pigmentation change & 0 & $1(8.3)$ & $1(5.6)$ & 0 & 0 & 0 \\
\hline Pruritus generalized & 0 & $1(8.3)$ & $1(5.6)$ & 0 & 0 & 0 \\
\hline Rash maculo-papular & 0 & 0 & 0 & $1(16.7)$ & 0 & 0 \\
\hline Urticaria & 0 & $1(8.3)$ & $1(5.6)$ & 0 & 0 & 0 \\
\hline $\begin{array}{l}\text { Injury, poisoning, and procedural } \\
\text { complications }\end{array}$ & 0 & $1(8.3)$ & $1(5.6)$ & 0 & 0 & 0 \\
\hline Contusion & 0 & $1(8.3)$ & $1(5.6)$ & 0 & 0 & 0 \\
\hline
\end{tabular}

Change from baseline in HBV DNA. A dose-dependent reduction from baseline in HBV DNA was observed at day 29 (7 d after the last dose and before initiation of NA therapy) in treatment-naïve patients who received bepirovirsen. The mean (s.d.) HBV DNA reduction from baseline to day 29 was $0.38(0.42) \log _{10} \mathrm{IU} \mathrm{ml} \mathrm{m}^{-1}$ $(P=0.116$ versus placebo $)$ and $1.66(1.48) \log _{10} \mathrm{IU} \mathrm{ml}^{-1}(P<0.001$ versus placebo) in the bepirovirsen $150 \mathrm{mg}$ and $300 \mathrm{mg}$ arms, respectively (Table 3). After day 29, HBV DNA levels declined substantially in all patients, as expected with the administration of NA treatment (Fig. 1b).
All six treatment-naïve patients who received bepirovirsen $300 \mathrm{mg}$ and had a $>1.0 \log _{10} \mathrm{IU} \mathrm{ml}^{-1}$ reduction in plasma HBsAg also had a $>0.5 \log _{10}$ IU ml ${ }^{-1}$ reduction in HBV DNA by day 29 ; with the three patients who experienced $\mathrm{a} \geq 3 \log _{10} \mathrm{IU} \mathrm{ml}^{-1}$ reduction in HBsAg also having a $>3 \log _{10} \mathrm{IU} \mathrm{ml}^{-1}$ reduction in HBV DNA by day 29 (Supplementary Fig. 6). One of the patients with a $>3.0 \log _{10} \mathrm{IU} \mathrm{ml}^{-1}$ reduction in HBV DNA had levels below the LLOQ $\left(20 \mathrm{IU} \mathrm{ml}^{-1}\right)$ by day 29, before initiation of tenofovir treatment. A fourth patient, who had a $0.98 \log _{10} \mathrm{IU} \mathrm{m}^{-1}$ reduction in plasma HBsAg at day 29, also had a $>3 \log _{10} \mathrm{IU} \mathrm{mm^{-1 }}$ reduction in HBV DNA. 
Table 3 | HBsAg and HBV DNA at baseline and day 29 in patients with CHB (full analysis population)

\begin{tabular}{|c|c|c|c|c|c|}
\hline & \multicolumn{3}{|c|}{ Treatment-naïve } & \multicolumn{2}{|c|}{ On-NA } \\
\hline & $\begin{array}{l}\text { Bepirovirsen } 150 \mathrm{mg} \\
\qquad(n=6)\end{array}$ & $\begin{array}{l}\text { Bepirovirsen } 300 \mathrm{mg} \\
\qquad(n=12)\end{array}$ & Placebo $(n=6)$ & $\begin{array}{l}\text { Bepirovirsen } 300 \mathrm{mg} \\
\quad(n=5)\end{array}$ & Placebo $(n=2)$ \\
\hline \multicolumn{6}{|c|}{ Baseline $\mathrm{HBsAg}\left(\log _{10} \mathrm{IU} \mathrm{ml^{-1 }}\right)$} \\
\hline$n$ & 6 & 12 & 6 & 5 & 2 \\
\hline Mean (s.d.) & $3.57(1.244)$ & $3.89(1.056)$ & $3.21(1.304)$ & $2.78(0.363)$ & $3.77(1.018)$ \\
\hline \multicolumn{6}{|c|}{ 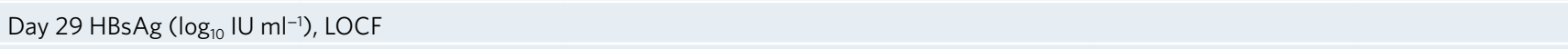 } \\
\hline Mean (s.d.) & $3.06(1.580)$ & $2.34(2.232)$ & $3.21(1.237)$ & $0.79(2.118)$ & $3.76(0.979)$ \\
\hline \multicolumn{6}{|c|}{ Change from baseline to day 29 in $\mathrm{HBsAg}\left(\log _{10} \mathrm{IU} \mathrm{ml^{-1 }}\right)$, LOCF } \\
\hline$n$ & 6 & 12 & 6 & 5 & 2 \\
\hline Mean (s.d.) & $-0.50(0.566)$ & $-1.56(1.379)$ & $0.00(0.112)$ & $-1.99(1.799)$ & $-0.01(0.039)$ \\
\hline$P$ value (versus placebo) & 0.245 & 0.001 & & 0.762 & \\
\hline \multicolumn{6}{|c|}{ Day 29 HBV DNA $\left(\log _{10} \mid \mathrm{IU} \mathrm{ml}^{-1}\right)$, LOCF } \\
\hline$n$ & 6 & 12 & 6 & 5 & 2 \\
\hline Mean (s.d.) & $7.03(1.451)$ & $5.12(3.073)$ & $5.57(2.429)$ & $1.37(0.167)$ & $<1.30(0.000)$ \\
\hline \multicolumn{6}{|c|}{ Change from baseline to day 29 in HBV DNA $\left(\log _{10} I U \mathrm{ml}^{-1}\right)$, LOCF } \\
\hline$n$ & 6 & 12 & 6 & 5 & 2 \\
\hline Mean (s.d.) & $-0.38(0.420)$ & $-1.66(1.479)$ & $0.00(0.471)$ & $0.08(0.167$ & $0.00(0.000)$ \\
\hline$P$ value (versus placebo) & 0.116 & $<0.001$ & & NA & \\
\hline
\end{tabular}

Baseline was the last nonmissing measurement before the first dose of the study drug. The last observation carried forward (LOCF) method was used to impute missing values. Comparison between bepirovirsen and pooled placebo was performed for each dose level separately using an analysis of covariance model with baseline as a covariate and treatment group as a factor. All comparisons were prespecified with no adjustment for multiple comparisons. Two-sided $P$ values are presented.

Although reductions in HBV DNA were observed in both $\mathrm{HBeAg}$-positive and $\mathrm{HBeAg}$-negative patients at day 29, the reduction was generally greater in HBeAg-negative patients. Baseline HBV DNA was substantially higher for HBeAg-positive patients, and fewer patients with higher DNA levels at day 29 had a reduction to the LLOQ at day 211 (Fig. 1b).

ALT increases. ALT flares, defined as an ALT level of $\geq 2 \times U L N$, were observed during this study. Of the 18 treatment-naïve patients administered bepirovirsen, eight experienced an ALT flare: two patients in the bepirovirsen $150 \mathrm{mg}$ arm and six in the bepirovirsen $300 \mathrm{mg}$ arm. Among these patients, both those in the $150 \mathrm{mg}$ arm and three of six patients in the $300 \mathrm{mg}$ arm had an ALT level $\geq 2 \times$ ULN at baseline, which further increased during treatment. In the on-NA group, all patients had a normal ALT level at baseline; however, three of five patients receiving bepirovirsen $300 \mathrm{mg}$ experienced an ALT flare. All ALT flares were transient and self-resolved. AST increases from baseline of at least two grades were observed in two treatment-naïve patients and two on-NA patients following treatment with bepirovirsen; these changes were concurrent with ALT increases but were of a smaller magnitude. There were no concurrent changes in bilirubin (total and direct) for any patient.

Reductions in HBsAg either preceded the ALT increases or occurred concomitantly with ALT flares (Fig. 3a-d and Supplementary Fig. 4). Generally, larger ALT increases (based on peak ALT) were associated with greater HBsAg reductions (Fig. $3 \mathrm{e}$ and Supplementary Fig. 6). For patients with a HBsAg reduction $\geq 3.0 \log _{10}$, ALT flares were observed for all on-NA patients (three) and for two of three treatment-naïve patients. In treatment-naïve patients, larger ALT increases were also associated with greater
HBV DNA reductions at day 29 (Supplementary Fig. 7). By contrast, no ALT flares were observed in patients without a HBsAg reduction $\left(<0.2 \log _{10}\right)$ or in patients receiving placebo. Comparison of ALT area under the curve (AUC) for days 1-113 by bepirovirsen dose level in patients with CHB and in healthy volunteers (unpublished data) when dosed for 4 weeks, shows ALT increases in the latter population were minor even at a higher dose than observed in patients with CHB (Fig. 3f).

The highest ALT concentration observed was $781 \mathrm{U} \mathrm{l}^{-1}$ (23.7× ULN; 26-fold change from baseline), which was recorded as a serious adverse event $15 \mathrm{~d}$ after the last dose of bepirovirsen $300 \mathrm{mg}$ (week 6) in a treatment-naïve patient (Fig. 3a and Supplementary Fig. 8a). Increased AST was also observed in this patient (up to $525 \mathrm{U} \mathrm{l}^{-1}$ at the same time point; 21 -fold change from baseline). ALT and AST levels decreased substantially within 2 weeks and returned to baseline by week 17 . The patient was asymptomatic throughout. Alkaline phosphatase elevation $(1.7 \times \mathrm{ULN})$ was also observed in this patient (Supplementary Fig. 8a); all other patients with ALT increases had no notable change in alkaline phosphatase. Another treatment-naïve patient in the bepirovirsen $300 \mathrm{mg}$ arm had an ALT flare $\geq 10 \times \mathrm{ULN}$, reaching a maximum ALT increase of $479 \mathrm{U} \mathrm{l}^{-1}$ at week 6 (approximately sevenfold change from baseline) (Supplementary Figs. 4d and $8 \mathrm{~b}$ ). This patient also had an associated increase in AST up to $343 \mathrm{U} \mathrm{l}^{-1}$ at week 6 (approximately sevenfold change from baseline). In the on-NA cohort, three patients experienced ALT flares, which were temporally associated with a reduction in HBsAg. These ALT increases were $<10 \times \mathrm{ULN}$, with maximum ALT levels $<300 \mathrm{IU} \mathrm{l}^{-1}$.

Change from baseline in the virological biomarkers $\mathrm{HBV}$ RNA, HBcrAg and HBeAg. Of the six treatment-naïve patients 


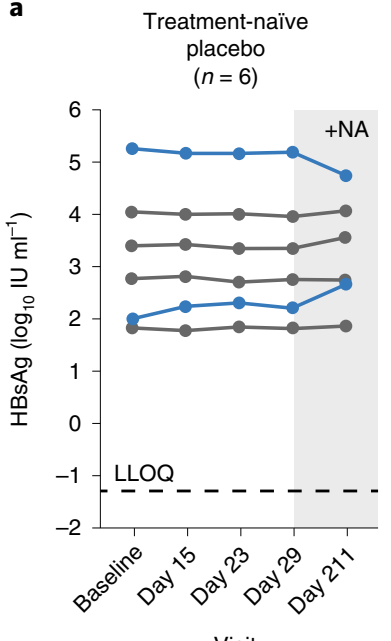

Visit

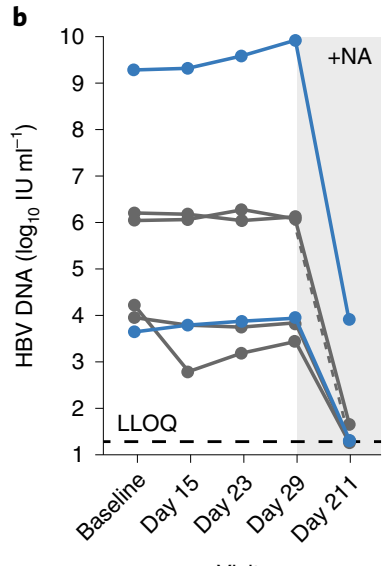

Visit
Treatment-naïve bepirovirsen $150 \mathrm{mg}$ $(n=6)$

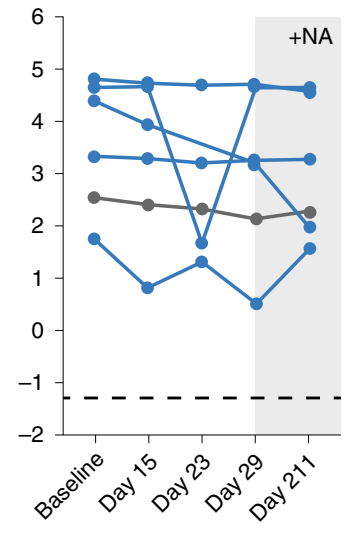

Visit

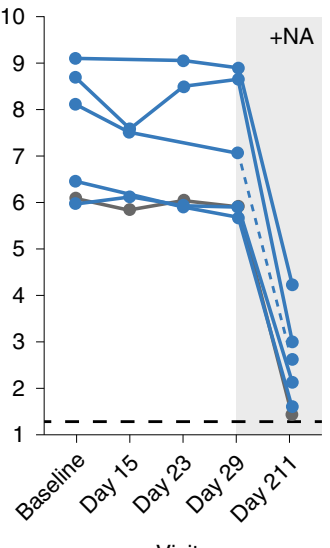

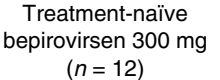

$(n=12)$

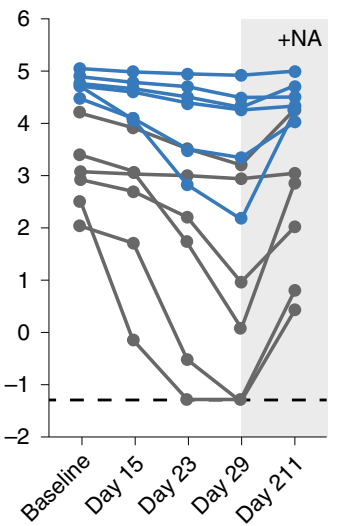

Visit

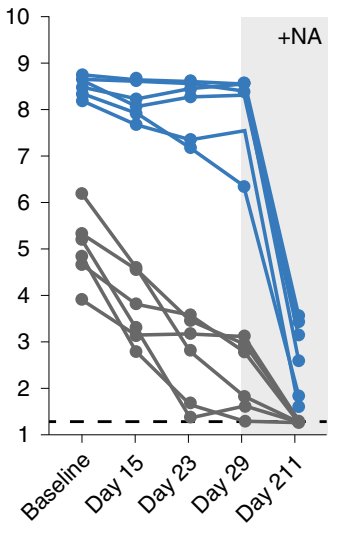

On-NA placebo $(n=2)$

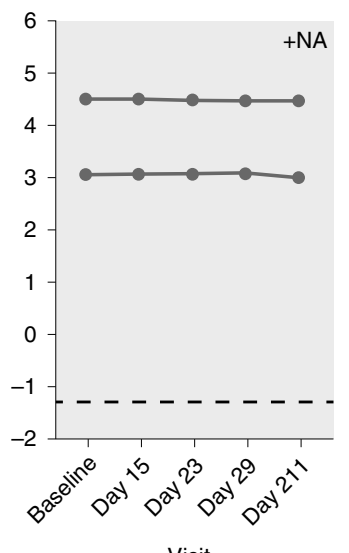

Visit

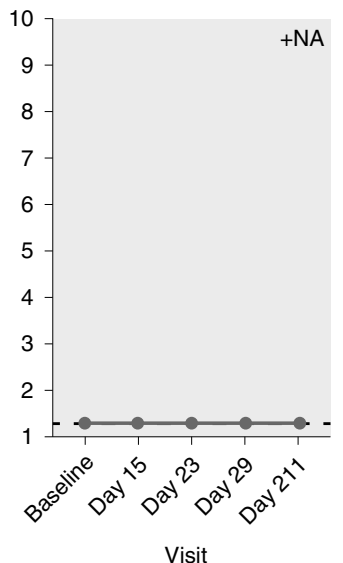

On-NA bepirovirsen $300 \mathrm{mg}$ $(n=5)$

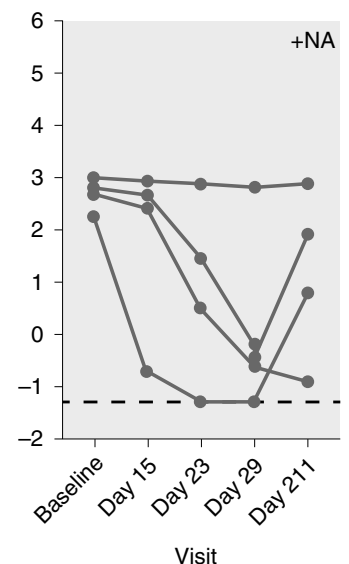

Visit

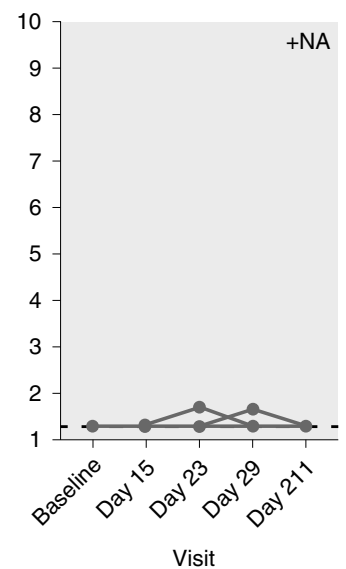

Fig. 1 | Change over time in serum HBsAg and HBV DNA. a,b, HBsAg (a) and HBV DNA (b) at baseline, day 15, day 23, day 29 and end of study (day 211) in patients with $\mathrm{CHB}$ (full analysis population). Blue lines indicate HBeAg-positive patients; gray lines indicate HBeAg-negative patients; black horizontal dashed line indicates LLOQ; gray shading indicates NA administration. In HBV DNA panels for treatment-naïve patients treated with placebo and bepirovirsen $150 \mathrm{mg}$, dashed lines between day 29 and day 211 are for patients whose day 211 test failed to produce a result. The last observation for these two patients (day 113) was carried forward to day 211. Four patients reached undetectable HBsAg levels; however, only three of the four reached LLOQ by day 29, the fourth patient reached LLOQ by day 36. Comparison between bepirovirsen and pooled placebo was performed for each dose level, separately, using an analysis of covariance model with baseline as a covariate and treatment group as a factor. For each comparison, if data departed substantially from normality, the Wilcoxon rank sum test was used.

receiving bepirovirsen $300 \mathrm{mg}$ and having a $\geq 1.0 \log _{10}$-transformed reduction in $\mathrm{HBsAg}\left(\mathrm{IU} \mathrm{ml}^{-1}\right)$ at day 29 , five and four, respectively, also had a concomitant $>0.5 \log _{10}$-transformed reduction by day 36 in HBV RNA (copies $\mathrm{ml}^{-1}$ ) and hepatitis B core-related antigen (HBcrAg; IU ml-1) (Supplementary Fig. 6). By contrast, both treatment-naïve patients receiving bepirovirsen $300 \mathrm{mg}$ and having $\leq 0.2 \log _{10}$-transformed reduction in $\mathrm{HBsAg}$, also had minimal change ( $\leq 0.2 \log _{10}$-transformed reduction) in HBV RNA and $\mathrm{HBcrAg}$ (Supplementary Fig. 6). Analysis was not possible in the on-NA patients because HBV RNA and HBcrAg at baseline were close to or below the LLOQ (as expected for patients receiving NA therapy). HBV RNA and $\mathrm{HBcrAg}$ responses over time are shown in Fig. 2.

In the 13 patients who were $\mathrm{HBeAg}$-positive at baseline, minimal reductions in serum $\mathrm{HBeAg}$ concentration (IU $\mathrm{ml}^{-1}$ ) were observed from baseline to day 29 and to week 31 (Supplementary Table 1). Reductions in HBeAg of the magnitude observed for HBsAg and HBV DNA were generally not observed, other than for the patient shown in Fig. $3 \mathrm{~d}$. In this patient, HBeAg was
$780 \mathrm{IU} \mathrm{ml}^{-1}$ at baseline and reduced by $1.0 \log _{10}$ on day $22,0.5 \log _{10}$ on day $29,0.9 \log _{10}$ on day $36,3.5 \log _{10}$ on day 113 and $3.0 \log _{10}$ on day 211 (Supplementary Fig. 9).

Seroconversion to anti-HBs or anti-HBe antibodies. Seroconversion was assessed as an exploratory objective. Transient treatmentemergent positivity for anti-HBs antibody, based on a qualitative test performed with the cut-off of $\geq 11.5 \mathrm{mIU} \mathrm{ml}^{-1}$, was observed for one of six and five of twelve treatment-naïve patients who received bepirovirsen $150 \mathrm{mg}$ and $300 \mathrm{mg}$, respectively, and for one of five on-NA patients who received bepirovirsen $300 \mathrm{mg}$. The treatment-naïve patient treated with $150 \mathrm{mg}$ was positive on day 22 and indeterminate on day 29 (Fig. 3d). The six patients treated with bepirovirsen $300 \mathrm{mg}$ were all positive on day 29; four treatment-naïve patients and one on-NA patient were positive and indeterminate, respectively, on day 57 (for examples, see Fig. 3a and Supplementary Fig. 4a,d). Two treatment-naïve patients and one on-NA patient with $\geq 3.0 \log _{10} \mathrm{IU} \mathrm{ml^{-1 }} \mathrm{HBsAg}$ reduction at day 29 experienced transient anti-HBs antibody positivity. 

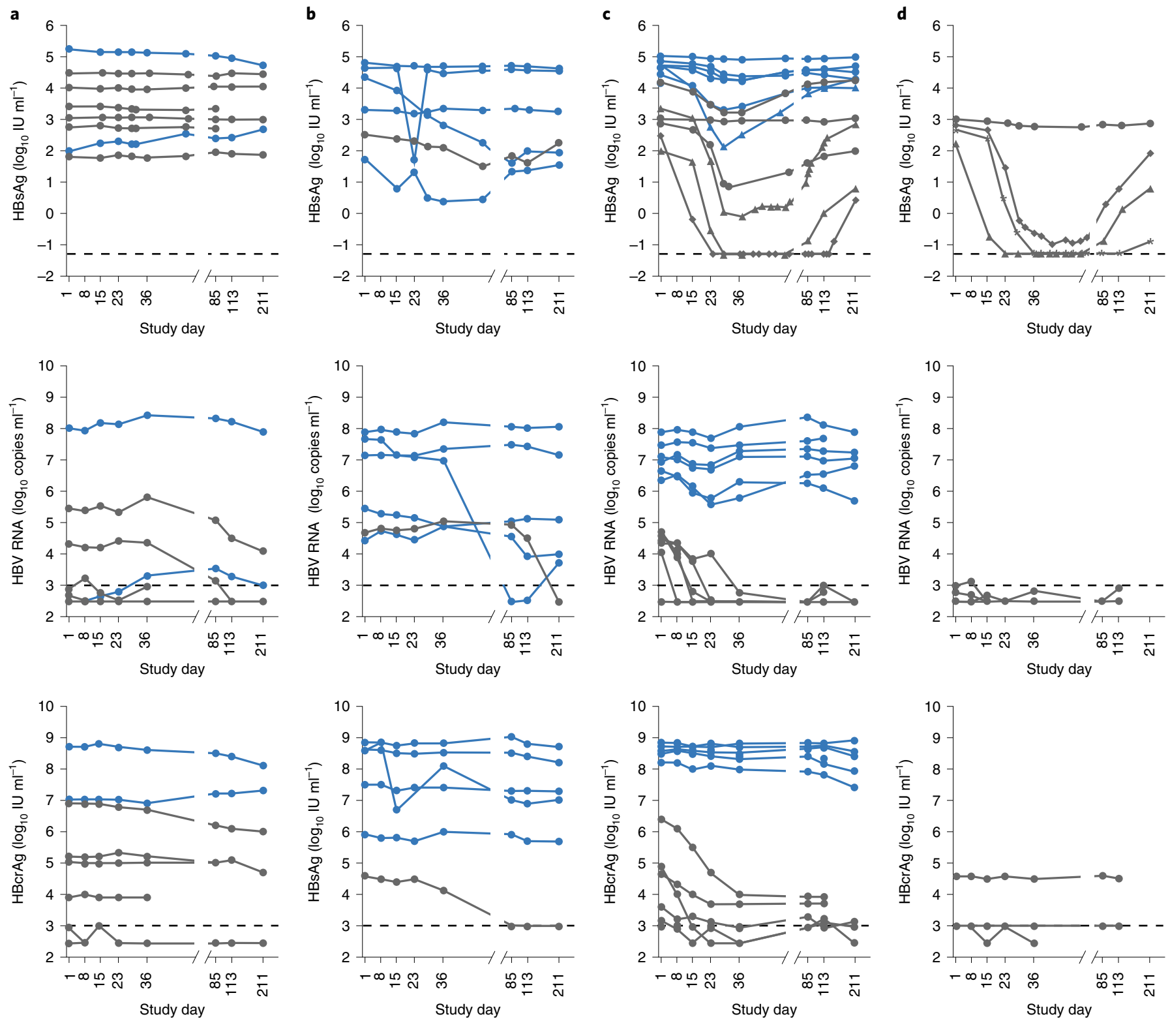

$\rightarrow$ HBeAg-positive

$\rightarrow-\mathrm{HBeAg-negative}$

Fig. 2 | Change in HBsAg, HBV RNA and HBcrAg (all time points). a, Placebo (NA-naïve and NA-treated; $n=8$ ). b, Bepirovirsen 150 mg (NA-naïve; $n=6$ ). c, Bepirovirsen $300 \mathrm{mg}$ (NA-naïve; $n=12$ ). d, Bepirovirsen $300 \mathrm{mg}$ (NA-treated; $n=4^{\star}$ ) (full analysis population; HBV RNA and HBcrAg analyses were post hoc). One patient discontinued treatment on study day 8 and is not shown. This patient discontinued treatment and withdrew from the study; as such they were not assessed beyond day 8 and are not shown in this figure (exclusion not prespecified), this patient was also excluded from the per-protocol population (according to prespecified criteria). Dotted lines denote LLOQ. The data shown are descriptive, no statistical analysis was conducted.

No patients who were anti-HBe negative at baseline, in either group, had anti-HBe antibody positivity at day 29. The treatment-naïve patient shown in Fig. 3d, who had a substantial and persistent reduction in $\mathrm{HBsAg}$, was negative for anti-HBe antibody from screening to day 29 but was positive from day 57 to the end of study (day 211), which coincides with the period when their $\mathrm{HBeAg}$ levels were $\leq 0.6 \log _{10}$ below baseline level. One patient in the on-NA group had detectable HBeAg at screening, day 1 and day 15, although they were considered $\mathrm{HBeAg}$ negative because all values were $<0.09 \mathrm{IU} \mathrm{ml}^{-1}$. From day 23, the patient's HBeAg levels were persistently below the LLOQ $\left(<0.06 \mathrm{IU} \mathrm{ml}^{-1}\right)$; however, on day 211 (final study visit) this patient tested positive for anti-HBe antibody.
Bepirovirsen binding site sequencing. The sequence of the bepirovirsen binding site was assessed via DNA or RNA sequencing (Supplementary Results and Supplementary Fig. 10). No sequence alterations were found in any of the samples assessed at baseline $(n=25)$, day $29(n=17)$ and day $113(n=7)$.

\section{Discussion}

This is the first study in which bepirovirsen has been administered to patients with CHB. A dose-dependent reduction in HBsAg and HBV DNA after 4 weeks of treatment with bepirovirsen (150 or $300 \mathrm{mg}$ ) was observed in treatment-naïve patients with $\mathrm{CHB}$, with a statistically significant reduction in HBsAg and HBV DNA compared 
a
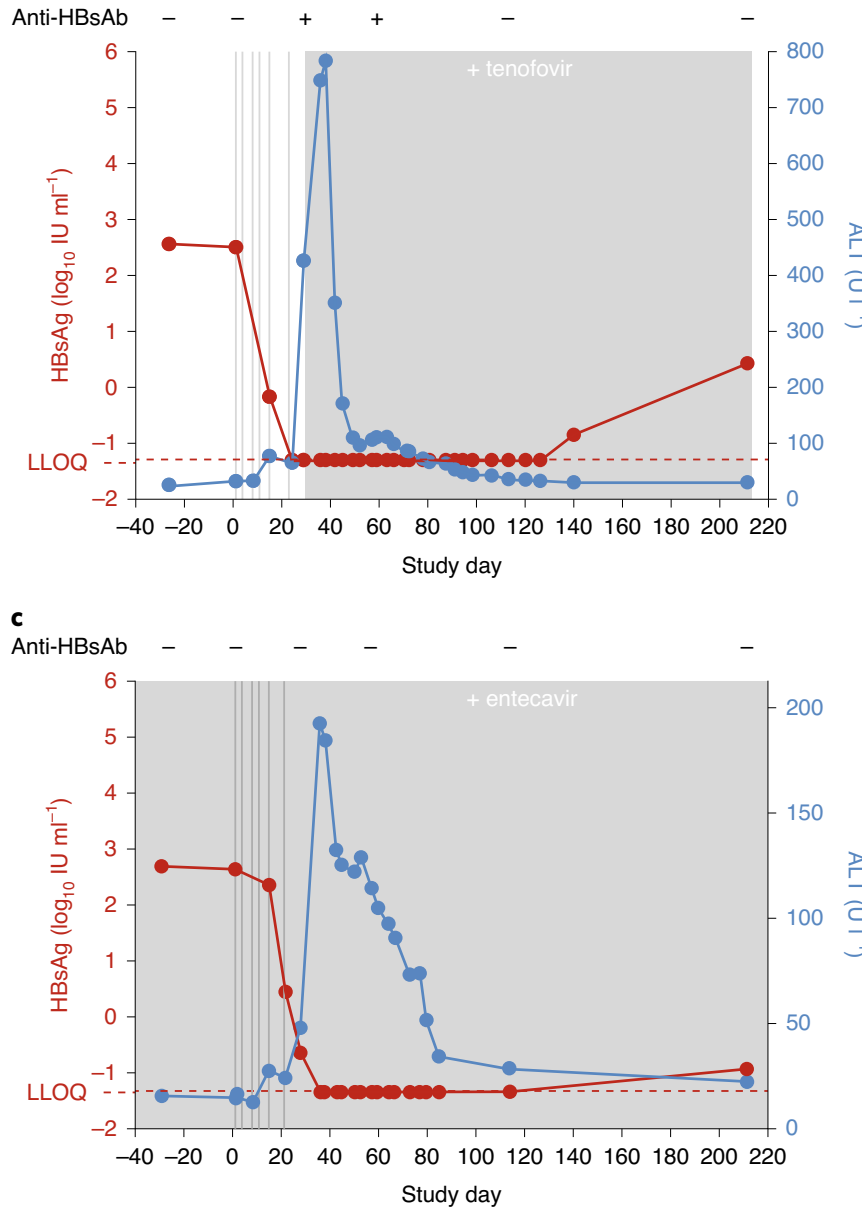

e

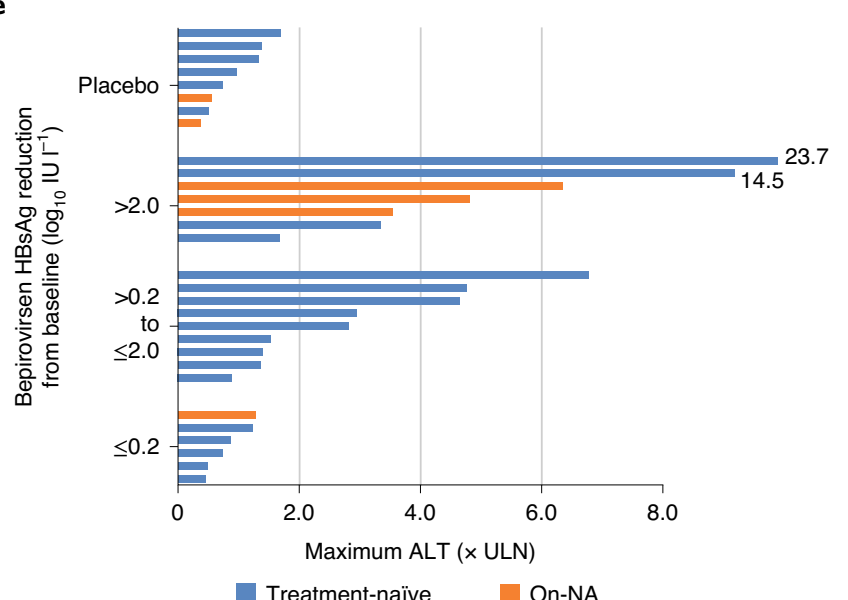

b

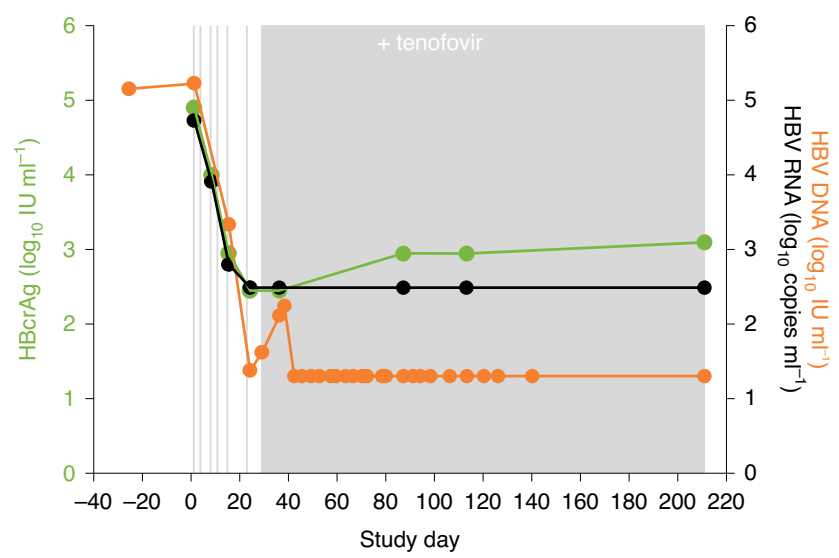

d

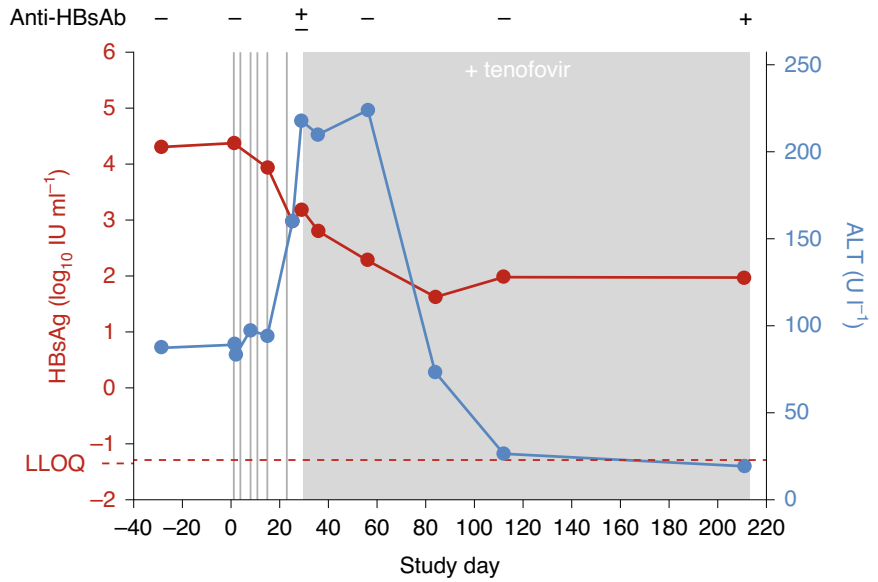

$\mathbf{f}$

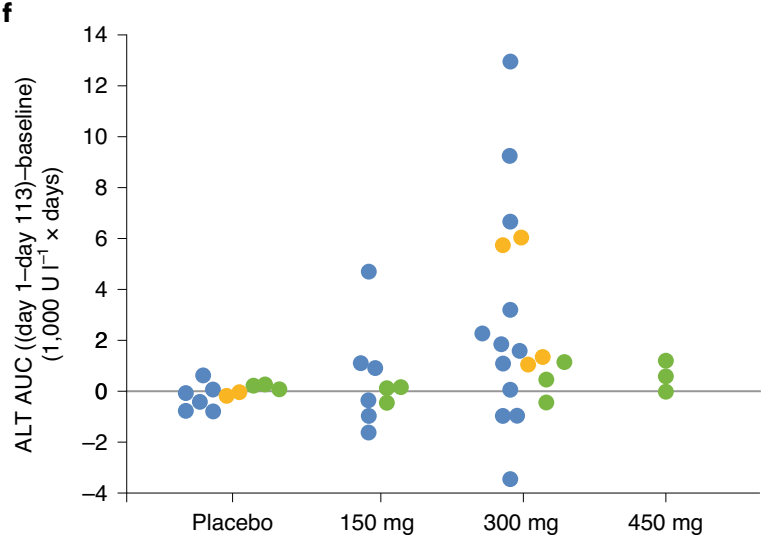

- Treatment-naïve Healthy volunteers On-NA

Fig. 3 | Profiles and relationships of ALT, HBsAg, HBcrAg and HBV DNA levels in treatment-naïve, NA-treated patients and healthy volunteers receiving different doses of bepirovirsen. a,b, HBsAg and ALT (a), and HBcrAg, HBV RNA and HBV DNA (b) in a treatment-naïve patient treated with bepirovirsen 300 mg. c, HBsAg and ALT in a patient already on entecavir treated with bepirovirsen 300 mg. d, HBsAg and ALT in a treatment-naïve patient treated with bepirovirsen $150 \mathrm{mg}$ (vertical lines indicate dose administration days; gray shading indicates NA dosing period for treatment-naïve patients). e, ALT results at day 29 categorized by HBsAg reduction from baseline. f, ALT AUC categorized by dose group in patients with CHB (safety population) and healthy volunteers (Study CS1 safety population; unpublished data) receiving bepirovirsen ( $y$ axis is the AUC of $A L T$ from day 1 to day 113 that is above the $A U C$ of baseline ALT maintained for the time period). +, positive for anti-HBsAb; -, negative for anti-HBsAb; \pm , indeterminate anti-HBsAb status; anti-HBsAg, antibody to HBV surface antigen. Data shown are descriptive, no statistical analysis was conducted.

with placebo in the bepirovirsen $300 \mathrm{mg}$ treatment group, but not in the bepirovirsen $150 \mathrm{mg}$ group. Reductions in HBsAg were also seen with bepirovirsen $300 \mathrm{mg}$ treatment in patients on stable NA therapy but were not statistically significant. However, the absolute mean change from baseline in patients treated with bepirovirsen $300 \mathrm{mg}$ was higher in the NA-treated group than in the 
treatment-naïve group, indicating that the lack of statistical significance may be due to the small sample size. Among the most robust responders, the HBsAg response was rapid and was prolonged after the end of treatment; however, the durability of response was variable. Given that HBsAg loss is a rare event with currently available treatments ${ }^{9}$, the possibility of a rapid and prolonged $\mathrm{HBsAg}$ response after only 4 weeks of bepirovirsen treatment supports further exploration of this dosing regimen and treatment duration in a larger number of patients to determine whether functional cure is possible. Additionally, the greatest HBsAg log declines were observed in patients with low baseline HBsAg levels, highlighting the importance of baseline HBsAg levels on HBsAg decline (at low baseline HBsAg values, an absolute HBsAg decrease was equivalent to a greater log decline).

$\mathrm{HBeAg}$-negative patients represent the majority of those with $\mathrm{CHB}$, and its prevalence has increased over the past decade ${ }^{1,16}$. HBeAg-negative patients tend to have lower HBV DNA levels and be in a later stage of infection than those who are HBeAg positive ${ }^{9}$. HBsAg reduction was observed in both HBeAg-negative and $\mathrm{HBeAg}$-positive patients treated with bepirovirsen $300 \mathrm{mg}$; however, greater $\log _{10}$-transformed reductions in $\mathrm{HBsAg}$ (and HBV DNA) were observed in $\mathrm{HBeAg-negative} \mathrm{patients.} \mathrm{Wooddell} \mathrm{et} \mathrm{al.} \mathrm{have}$ previously demonstrated that a substantial proportion of HBsAg in $\mathrm{HBeAg-negative} \mathrm{patients} \mathrm{is} \mathrm{derived} \mathrm{from} \mathrm{integrated} \mathrm{HBV}$ sequences and integration can lead to the loss of target sequences in the HBV mRNA $^{15}$. The substantial reduction of HBsAg in HBeAg-negative patients suggests that the bepirovirsen target site is preserved in the majority of transcripts derived from integrated HBV genomes.

Compared with $\mathrm{HBeAg}$-positive patients, HBeAg-negative patients had lower baseline HBsAg and HBV DNA, which may be a factor responsible for the improved response. These findings suggest that high baseline antigen and viral loads may be factors contributing negatively to the response. The observation that a higher viral load at day 29 appeared to be a negative factor for achieving a reduction in HBV DNA with NA treatment to below LLOQ at day 211 supports this possibility. Most $\mathrm{HBeAg}$-positive patients also showed a minimal reduction in $\mathrm{HBeAg}$ after treatment, except for one patient in the bepirovirsen $150 \mathrm{mg}$ group, who had a substantial $\mathrm{HBeAg}$ reduction (3.0 $\log _{10}$ at day 211) following an ALT flare. Given that reductions in absolute HBsAg levels were observed in some $\mathrm{HBeAg}$-positive patients with high baseline $\mathrm{HBsAg}$, exploration of a longer treatment duration may improve the response in $\mathrm{HBeAg}$-positive patients.

Three patients treated with bepirovirsen $300 \mathrm{mg}$ had a minimal response with $<0.2 \log _{10}$-transformed reduction in HBsAg. The first patient was treatment-naïve and $\mathrm{HBeAg}$ positive, with a high baseline HBsAg $\left(5.04 \log _{10} \mathrm{IU} \mathrm{ml}^{-1}\right)$. Although only achieving $<0.2 \log _{10}$-transformed reduction, HBsAg levels decreased from $\sim 110,000 \mathrm{IU} \mathrm{ml}^{-1}$ at day 1 to $\sim 81,000 \mathrm{IU} \mathrm{ml}^{-1}$ at day 36 . It is possible that the duration of exposure to bepirovirsen was not long enough to elicit a robust response. The second and third patients were treatment-naïve and on-NA treatment, respectively. Both were $\mathrm{HBeAg}$ negative, and both had a baseline HBsAg level similar to patients with a robust response, indicating that baseline $\mathrm{HBsAg}$ levels do not solely explain the lack of response. Additionally, sequencing of samples from both patients confirmed the presence of the bepirovirsen binding site, indicating that the less robust $\mathrm{HBsAg}$ decrease is not due to loss of the target site. Despite the minimal reduction in $\mathrm{HBs} A g$, the treatment-naïve patient had a -0.81 $\log _{10}$-transformed reduction in HBV DNA at day 29, indicating that HBV in this patient was possibly susceptible to bepirovirsen; however, this patient's HBV DNA levels were already declining from screening. The HBV RNA and HBcrAg were both at the LLOQ at baseline and throughout the study. The on-NA patient had detectable HBcrAg levels at baseline, which did not change on treatment; HBV DNA and RNA were not detectable at baseline. Study of bepirovirsen treatment of longer duration is needed to determine whether the HBsAg levels in such HBeAg-negative patients can be further reduced.

Previously, Wooddell et al. proposed that the less-efficient HBsAg reduction following treatment with a small interfering RNA in $\mathrm{HBeAg}$-negative HBV-infected chimpanzees was due to loss of the target binding site in mRNA derived from integrated HBV genetic material; the bepirovirsen binding site is $~ 200$ nucleotides upstream of the integration site identified by Wooddell et al. ${ }^{15}$. In this study, three on-NA patients (all HBeAg negative) experienced reductions in $\mathrm{HBsAg} \geq 3.0 \log _{10} \mathrm{IU} \mathrm{ml}^{-1}$. Baseline levels of $\mathrm{HBcrAg}$ and $\mathrm{HBV}$ RNA were at or near to the limit of assay sensitivity, which limits interpretation, but implies that low levels of covalently closed circular HBV DNA transcriptional activity are present and most HBsAg is likely derived from the integrated DNA. This suggests that bepirovirsen can reduce transcripts derived from integrated HBV DNA.

As expected, HBV DNA levels declined substantially in treatment-naïve patients after administration of NA treatment on day 29. These reductions were similar across the placebo, bepirovirsen $150 \mathrm{mg}$ and $300 \mathrm{mg}$ groups, which suggests that bepirovirsen treatment does not affect response to NAs. Furthermore, the increases in HBsAg after day 29, alongside the reductions in HBV DNA, confirm that NA treatment does not directly reduce HBsAg. However, we cannot exclude the possibility of a subtle effect early in NA treatment that may have contributed to further small reductions in HBsAg in the weeks immediately following day 29 in some patients.

Changes in ALT were assessed due to the known temporal link to HBsAg clearance, wherein ALT flares during NA therapy were associated with subsequent $\mathrm{HBsAg}$ decline and $\mathrm{HBsAg} \operatorname{loss}^{17}$. In the current study, HBsAg reduction either preceded or occurred in parallel with the ALT increase. The temporal profile observed in this study is different from that shown in a phase 3 study of HBeAg-positive patients treated with NA in which ALT flare occurred first, with HBsAg loss occurring a median of 56 weeks later ${ }^{17}$. The difference may be due to the differing mechanisms of action. It was hypothesized that ALT flare following NA treatment achieved HBV control, with eventual HBsAg loss occurring via hepatocyte turnover ${ }^{17}$. Based on the findings of the current study, we suggest that the observed HBsAg reduction is related to bepirovirsen treatment, with the reduction in HBsAg leading to ALT increase, which is possibly due to immune clearance of infected hepatocytes. ALT increases were also observed in three on-NA patients in whom HBV DNA was suppressed already. Therefore, it is unlikely that there is a connection between HBV DNA reduction and ALT increase.

Eleven of 23 patients with $\mathrm{CHB}$ who received bepirovirsen experienced a maximum ALT $>2 \times$ ULN during this study. By contrast, none of the 12 healthy volunteers in the phase 1 study who received a single dose of bepirovirsen $300 \mathrm{mg}$ and only one of nine healthy volunteers who received bepirovirsen $300 \mathrm{mg}$ for 4 weeks reported ALT increases $\geq 2 \times U L N$; the patient in the bepirovirsen $300 \mathrm{mg}$ arm had two ALT elevations that reached 2.6× ULN (unpublished data). Furthermore, no ALT elevations were observed in patients without a HBsAg reduction. Taken together, these results are more consistent with ALT increase as a pharmacological response to bepirovirsen targeting HBV RNA than with ALT as a toxicity from bepirovirsen exposure. However, given the small safety database, further characterization of ALT flares is warranted in future studies to further evaluate this hypothesis and rule out the possibility of drug-induced liver injury. Future studies should also consider the potential for grade 4 ALT flares in patients with cirrhosis, who were excluded in this study. A cautious approach is needed in these patients to minimize the risk from ALT elevations in patients with impaired hepatic reserve.

The observed transient appearance of anti-HBs antibody in some patients is consistent with the continuous synthesis of 
antibody that is sequestered into immune complexes by a large excess of circulating HBsAg. Reducing HBsAg via treatment with bepirovirsen changes this equilibrium so that free antibody appears in the circulation; the gradual increases in HBsAg observed in most patients after the end of bepirovirsen treatment may reduce circulating anti-HBs antibodies again via sequestration.

Bepirovirsen showed an acceptable safety and tolerability profile in patients with CHB. It is well established that adverse events (AEs) can lead to low clinical adherence, and this is an important consideration given that the participants receiving bepirovirsen experienced more AEs of fever and injection site conditions. However, these events were mostly grade 1 , and did not require dose interruptions. Given the generally mild nature of these AEs, and the overall safety profile, bepirovirsen was considered suitable for continued clinical study; however, longer studies are required to provide more insight into the tolerability of and adherence to bepirovirsen when administered for longer periods. There were no serious AEs other than the ALT increases discussed above. CRP elevations were observed in patients with $\mathrm{CHB}$, which is in line with previous studies of phosphorothioate oligonucleotides ${ }^{18-20}$, suggesting that it may be a class effect. Complement activation is an established class effect in nonhuman primates, although this has not translated into humans ${ }^{21}$; the lack of complement activation related to bepirovirsen is consistent with previous clinical studies of phosphorothioate oligonucleotides. Similarly, dose-related, transient activated partial thromboplastin time prolongations following administration of bepirovirsen were observed and are a known class effect of phosphorothioate oligonucleotides ${ }^{22,23}$.

The limitations of this study include small patient numbers and the short duration of bepirovirsen treatment, which preclude a conclusion of whether bepirovirsen can achieve functional cure. Although the $150 \mathrm{mg}$ dose did not result in a statistically significant reduction in HBsAg, it merits consideration as part of a consolidation regimen. Additionally, immunological investigations will be important to evaluate the hypothesis of immune restoration indicated by ALT flares associated with HBsAg reductions. Furthermore, the study did not provide insight into effects in the liver due to the absence of liver biopsies.

In summary, this study suggests that bepirovirsen can induce rapid and prolonged reductions in $\mathrm{HBsAg}$ in patients with $\mathrm{CHB}$, both treatment-naive patients and those on stable NA therapy. Combined with a favorable safety profile, these preliminary findings warrant further investigation into the dose and duration of bepirovirsen in a larger population of patients with CHB.

\section{Online content}

Any methods, additional references, Nature Research reporting summaries, source data, extended data, supplementary information, acknowledgements, peer review information; details of author contributions and competing interests; and statements of data and code availability are available at https://doi.org/10.1038/ s41591-021-01513-4.

Received: 8 March 2021; Accepted: 18 August 2021; Published online: 12 October 2021

\section{References}

1. Guidelines for the Prevention, Care and Treatment of Persons with Chronic Hepatitis B Infection (World Health Organization, 2015); https://www.who.int/ hiv/pub/hepatitis/ hepatitis-b-guidelines/en/

2. Polaris Observatory Collaborators. Global prevalence, treatment, and prevention of hepatitis B virus infection in 2016: a modelling study. Lancet Gastroenterol. Hepatol. 3, 383-403 (2018).
3. Cornberg, M., Lok, A. S., Terrault, N. A. \& Zoulim, F. Guidance for design and endpoints of clinical trials in chronic hepatitis B - report from the 2019 EASLAASLD HBV Treatment Endpoints Conference. J. Hepatol. 72, 539-557 (2020).

4. Kim, G. A. et al. HBsAg seroclearance after nucleoside analogue therapy in patients with chronic hepatitis B: clinical outcomes and durability. Gut 63, 1325-1332 (2014)

5. Lok, A. S., Zoulim, F., Dusheiko, G. \& Ghany, M. G. Hepatitis B cure: from discovery to regulatory approval. Hepatology 66, 1296-1313 (2017).

6. Yuen, M. F. et al. HBsAg seroclearance in chronic hepatitis B in Asian patients: replicative level and risk of hepatocellular carcinoma. Gastroenterology 135, 1192-1199 (2008).

7. Bertoletti, A. \& Ferrari, C. Innate and adaptive immune responses in chronic hepatitis B virus infections: towards restoration of immune control of viral infection. Gut 61, 1754-1764 (2012).

8. Tan, A., Koh, S. \& Bertoletti, A. Immune response in hepatitis B virus infection. Cold Spring Harb. Perspect. Med 5, a021428 (2015).

9. European Association for the Study of the Liver. EASL 2017 Clinical Practice Guidelines on the management of hepatitis B virus infection. J. Hepatol. 67, 370-398 (2017).

10. Lam, Y. F. et al. Seven-year treatment outcome of entecavir in a real-world cohort: effects on clinical parameters, HBsAg and HBcrAg levels. Clin. Transl. Gastroenterol. 8, e125 (2017).

11. Seto, W. K. et al. Patterns of hepatitis B surface antigen decline and HBV DNA suppression in Asian treatment-experienced chronic hepatitis B patients after three years of tenofovir treatment. J. Hepatol. 59, 709-716 (2013).

12. Wong, D. K. et al. Reduction of hepatitis B surface antigen and covalently closed circular DNA by nucleos(t)ide analogues of different potency. Clin. Gastroenterol. Hepatol. 11, 1004-1010.e1 (2013).

13. Swayze, E. E., Freler, M. M., McCaleb, M. L. \& Zhang, H. Modulation of hepatitis B virus (HBV) expression. Google Patents https://patents.google. com/patent/US8642752B2 (2014).

14. Billioud, G. et al. In vivo reduction of hepatitis B virus antigenemia and viremia by antisense oligonucleotides. J. Hepatol. 64, 781-789 (2016).

15. Wooddell, C. I. et al. RNAi-based treatment of chronically infected patients and chimpanzees reveals that integrated hepatitis B virus DNA is a source of HBsAg. Sci. Transl. Med. 9, eaan0241 (2017).

16. Funk, M. L., Rosenberg, D. M. \& Lok, A. S. World-wide epidemiology of HBeAg-negative chronic hepatitis B and associated precore and core promoter variants. J. Viral Hepat. 9, 52-61 (2002).

17. Wong, D. et al. ALT flares during nucleotide analogue therapy are associated with HBsAg loss in genotype A HBeAg-positive chronic hepatitis B. Liver Int. 38, 1760-1769 (2018).

18. Ackermann, E. J. et al. Suppressing transthyretin production in mice, monkeys and humans using 2nd-generation antisense oligonucleotides. Amyloid 23, 148-157 (2016).

19. Flaim, J. D., Grundy, J. S., Baker, B. F., McGowan, M. P. \& Kastelein, J. J. Changes in mipomersen dosing regimen provide similar exposure with improved tolerability in randomized placebo-controlled study of healthy volunteers. J. Am. Heart Assoc. 3, e000560 (2014).

20. Graham, M. J. et al. Antisense oligonucleotide inhibition of apolipoprotein C-III reduces plasma triglycerides in rodents, nonhuman primates, and humans. Circ. Res. 112, 1479-1490 (2013).

21. Shen, L. et al. Mechanistic understanding for the greater sensitivity of monkeys to antisense oligonucleotide-mediated complement activation compared with humans. J. Pharmacol. Exp. Ther. 351, 709-717 (2014).

22. Sheehan, J. P. \& Lan, H. C. Phosphorothioate oligonucleotides inhibit the intrinsic tenase complex. Blood 92, 1617-1625 (1998).

23. Sheehan, J. P. \& Phan, T. M. Phosphorothioate oligonucleotides inhibit the intrinsic tenase complex by an allosteric mechanism. Biochemistry 40, 4980-4989 (2001).

Publisher's note Springer Nature remains neutral with regard to jurisdictional claims in published maps and institutional affiliations.

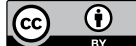

Open Access This article is licensed under a Creative Commons Attribution 4.0 International License, which permits use, sharing, adaptation, distribution and reproduction in any medium or format, as long as you give appropriate credit to the original author(s) and the source, provide a link to the Creative Commons license, and indicate if changes were made. The images or other third party material in this article are included in the article's Creative Commons license, unless indicated otherwise in a credit line to the material. If material is not included in the article's Creative Commons license and your intended use is not permitted by statutory regulation or exceeds the permitted use, you will need to obtain permission directly from the copyright holder. To view a copy of this license, visit http://creativecommons.org/licenses/by/4.0/. (c) The Author(s) 2021
} 


\section{Methods}

Study design. The study was approved by an independent ethics committee or institutional review board (IRB): IRB of the University of Hong Kong/ Hospital Authority Hong Kong West Cluster, Queen Mary Hospital, Hong Kong; Seoul National University Hospital IRB, Republic of Korea; Kyungpook National University IRB, Republic of Korea; Seoul St. Mary's Hospital IRB Republic of Korea; Pusan National University Hospital IRB, Republic of Korea; Korea University Ansan Hospital IRB, Republic of Korea; Inje University Busan Paik Hospital IRB Republic of Korea. The study was conducted in accordance with the Declaration of Helsinki (https://www. wma.net/policies-post/wma- declaration-of-helsinki-ethical-principles-formedical-research-involving-human-subjects/) and International Conference on Harmonisation Good Clinical Practice (https://ichgcp.net/). An independent monitoring committee oversaw the study. All patients provided written informed consent to participate in the trial before any study-specific procedures; patients were not compensated for participation in the study, but where permitted by local regulations and ethics committees, reasonable expenses were reimbursed.

The trial was composed of a double-blinded, randomized, placebo-controlled, dose-escalation study in treatment-naïve patients $(n=24)$ and an add-on exploratory cohort of patients already receiving stable NA regimens (on-NA patients; $n=7$ ) (Supplementary Fig. 1). Patients were enrolled from one center in Hong Kong and five centers in the Republic of Korea. The first patient was enrolled on 22 February 2017, the last patient was enrolled on 30 April 2019, the last patient visit was on 18 December 2019 and the study was completed on 19 December 2019. Patients were identified by the investigators or enquired about the study after reading patient information materials. The demography of the patients is broadly consistent with the HBV patient population.

The rationale for the weekly dosing frequency was supported by prior preclinical and clinical evaluations of more than 15 other $2^{\prime}$-MOE chimeric ASOs administered during preclinical and clinical studies ${ }^{24,25}$. All these $2^{\prime}$-MOE chimeric ASOs have very similar physico-chemical properties, because they have largely similar sugar-phosphorothioate-sugar backbones, and vary mainly in the order of the four bases ${ }^{24,25}$. Twice weekly dosing in weeks 1 and 2 constituted loading doses, with the aim of reaching steady-state hepatic concentrations following the week 3 dose (that is, before the last dose in the schedule) instead of 13-15 weeks without loading. The main assessments of single-agent treatment effects of bepirovirsen were performed on day 29, $7 \mathrm{~d}$ after the last dose of study drug (bepirovirsen or placebo). After these assessments, all patients received daily treatment with NA to end of study (week 31; day 211). Patients already on a stable NA regimen (on-NA) received treatment throughout (during all study periods).

Objectives and endpoints. The primary objective of the study was to examine the safety and tolerability of bepirovirsen administration in treatment-naïve participants with $\mathrm{CHB}$ infection (primary endpoints were AEs, clinical laboratory tests, vital signs and body weight, physical examination, electrocardiogram and concomitant medication usage)

Secondary objectives and endpoints were to: examine the effects of bepirovirsen administration on plasma HBV DNA concentration (change from baseline to day 29 and week 31); examine the effects of bepirovirsen administration on serum HBsAg concentration (change from baseline to day 29 and week 31, proportion of participants with HBsAg loss at day 29 and at week 31); examine the effect of bepirovirsen administration on serum HBeAg concentration in patients who were $\mathrm{HBeAg}$ positive at baseline (change from baseline to day 29 and week 31 , proportion of participants with $\mathrm{HBeAg}$ loss at day 29 and at week 31 ); assess plasma pharmacokinetics of bepirovirsen in patients with chronic HBV infection (to be published separately); and describe the safety and tolerability of tenofovir disoproxil fumarate (TDF) (and entecavir (ETV) if administered) therapy following conclusion of bepirovirsen administration (AEs after day 29; as the safety profile of TDF and ETV is well established we have not reported this endpoint here).

Exploratory endpoints and objectives included describing the rate of seroconversion to anti-HBs or anti-HBe antibody-positive during treatment with bepirovirsen and then during subsequent treatment with TDF, or ETV if administered (proportion of patients with antibody positivity at day 29 and at week 31 ).

Key eligibility criteria. Inclusion criteria. Inclusion criteria were chronic $\mathrm{HBV}$ infection $\geq 6$ months and serum $\mathrm{HBsAg} \geq 50 \mathrm{IU} \mathrm{ml}^{-1}$; both $\mathrm{HBeAg}$-positive and $\mathrm{HBeAg}$-negative patients could participate. Treatment-naïve patients had a plasma HBV DNA $\geq 2 \times 10^{3} \mathrm{IU} \mathrm{ml}^{-1}$. On-NA patients had HBV DNA adequately suppressed (plasma or serum HBV DNA below LLOQ (20 IU ml-1)), were taking stable TDF or ETV for $\geq 12$ months and expected to continue taking stable TDF or ETV without change through to the end of their participation in this study.

Exclusion criteria. Exclusion criteria were: a history of liver cirrhosis and/or evidence of cirrhosis, liver failure, liver disease other than hepatitis B, Gilbert's syndrome or history of laboratory results consistent with Gilbert's syndrome, extrahepatic disorders possibly related to HBV immune complexes, excess alcohol consumption; co-infection with hepatitis C virus, hepatitis D virus or HIV; screening laboratory values of ALT and AST $>5 \times$ ULN. Treatment-naïve patients were in current or prior receipt of anti-HBV NA therapy. Patients who had failed prior interferon treatment more than 6 months before screening may be evaluated for possible participation in the study.

Full eligibility criteria are listed in Supplementary Methods.

Treatment and follow-up schedule. This study was composed of a double-blinded, randomized, placebo-controlled, dose-escalation study in treatment-naïve patients with CHB. Escalation to the next dose level required Data and Safety Monitoring Board approval. Dose escalation and study enrollment ceased in the event of death of one patient or the occurrence of the same dose-limiting toxicity in two patients within one dose cohort. Dose-limiting toxicity was defined as AEs possibly related or related to study drug administration that met the following criteria: confirmed laboratory result meeting one of the stopping criteria (Supplementary Methods); all DAIDS grade $\geq 3$ nonlaboratory AEs with the exception of influenza-like symptoms consistent with the typical acute, transient, responses to 2'-MOE-modified chimeric phosphorothioate ASO injection; or any DAIDS grade 4 confirmed laboratory AE (other than those included in the stopping criteria; Supplementary Methods).

Although the Data and Safety Monitoring Board supported dose escalation to $450 \mathrm{mg}$, it was acknowledged that further evaluation of the $300 \mathrm{mg}$ dose was warranted and the sponsor continued evaluation of the $300 \mathrm{mg}$ dose in the third cohort to allow further characterization of the antiviral effect of bepirovirsen at the $300 \mathrm{mg}$ dose level. One exploratory cohort was added to evaluate add-on treatment with bepirovirsen $300 \mathrm{mg}$ versus placebo in on-NA patients with $\mathrm{CHB}$. Approximately eight patients were planned. The study design is illustrated in Supplementary Fig. 1.

Patients were randomized ( $3: 1$ within each dose cohort) to bepirovirsen or placebo according to the randomization schedule (permuted block). The investigator (or designee) obtained the unique study treatment number via an interactive voice/internet response system. Six doses of bepirovirsen or placebo were administered via subcutaneous injection on days $1,4,8,11,15$ and 22; patients were followed until day 211 . On day 29 , the effects of treatment were assessed. The endpoint assessment for antiviral activity was on day 29. TDF (or ETV) treatment was initiated on day 29 after HBV measurements in the treatment-naïve cohort.

All participants, study monitors, study center personnel and contract research organization personnel were blinded to treatment assignment. A global protocol amendment was made to include an on-NA cohort, in which participants with $\mathrm{CHB}$ already on a stable regimen of TDF or ETV were treated with $300 \mathrm{mg}$ of bepirovirsen by the same dosing schedule as treatment-naïve patients; all applicable sections of the protocol (including inclusion and exclusion criteria) were updated to reflect the addition of the on-NA cohort. The amendment, implemented after study initiation and demonstration of the drug's antiviral effect in treatment naïve patients, was introduced to determine whether antiviral effects could be observed in patients receiving stable NA therapy. The $300 \mathrm{mg}$ dose of bepirovirsen was selected for this cohort based on the data from the treatment-naïve patient cohort. All other protocol amendments and changes to study conduct or planned analyses are described in Supplementary Methods. All protocol amendments were approved by the designated IRB for each study site (Methods).

Assessments. Data were collected from 22 February 2017 to 19 December 2019. Blood samples for quantitative $\mathrm{HBsAg}, \mathrm{HBeAg}$ and $\mathrm{HBV}$ DNA measurement were collected at screening, pre-dose on days 1,15 and 29, and any time on days $23,36,57,85,113$ and 211. Blood samples for categorical anti-HBs and anti-HBe antibodies measurement were collected at screening, pre-dose on days 1 and 29, and any time on days 57, 113 and 211.

Virology assessments included quantitative measurement of serum HBsAg (COBAS HBsAg quant II; LLOQ: $0.05 \mathrm{IU} \mathrm{ml}^{-1}$ (Roche)), serum HBV DNA (COBAS Ampliprep/COBAS Taqman HBV test v.2.0 (Roche); LLOQ: $20 \mathrm{IU} \mathrm{ml}^{-1}$ ), serum HBeAg (LIAISON quantitative hepatitis B antigen by electrochemiluminescence immunoassay (DiaSorin)), serum HBcrAg (Lumipulse chemiluminescent enzyme immunoassay (Fujirebio), which utilized the Lumipulse G series system; LLOQ: $3.0 \log _{10} \mathrm{U} \mathrm{ml}^{-1}$ ), HBs antibodies (COBAS Elecsys anti-HBs II (Roche)), and HBe antibodies (COBAS Elecsys anti-HBe ECLIA (Roche)). Levels of HBV RNA were assayed by quantitative real-time polymerase chain reaction in the Applied Biosystems 7900HT Fast Real-Time PCR System with a TaqMan probe method (LLOQ: 10,862 copies $\mathrm{ml}^{-1}$ ). Population sequencing of HBV DNA or RNA was carried out on samples collected at baseline, end of bepirovirsen treatment period (day 29) and at day 113.

$\mathrm{HBs} \mathrm{g}$ and $\mathrm{HBeAg}$ quantitation were performed by PPD laboratories. HBV RNA quantitation was performed at DDL Diagnostic Laboratory. DNA and RNA sequencing were performed by WuXi AppTec to detect the presence of the bepirovirsen binding site sequence (GCACTTCGCTTCACCTCTGC).

Statistical methods. The enrolled population included all patients who signed the informed consent form. The safety population included all randomized patients who received one or more doses of bepirovirsen or placebo and was used for all safety analyses. The full analysis population (representing the practically feasible intent-to-treat population) included patients from the safety population with baseline and one or more post-baseline plasma HBV DNA concentrations. The 
per-protocol population included patients in the full analysis set who received at least five doses of bepirovirsen or placebo during the 4-week treatment period, had plasma HBV DNA concentration measured at day 29, and had no significant protocol deviations that would be expected to affect efficacy assessments. The full analysis and per protocol populations were used for efficacy endpoints.

There is no statistical rationale for the selected sample size of eight participants per cohort. The sample size was based on prior experience to ensure adequate initial assessment of the safety and tolerability of bepirovirsen while minimizing the number of patients unnecessarily exposed to the drug. The protocol included the ability to repeat a cohort if additional data at a specified cohort was warranted. No power calculation was performed.

No formal interim analysis was planned. However, an unblinded interim analysis for treatment-naïve dose cohorts was performed after all treatment-naïve patients had completed their day 29 assessments. An unblinded interim analysis for on-NA patients was performed after all on-NA patients had completed their day 29 assessments.

SAS v.9.4 was used for data analyses in this study. Demographic and baseline characteristics, and efficacy endpoints were summarized descriptively. Safety analyses were conducted on the safety population and summarized by treatment group. There were no formal hypotheses; efficacy analyses for comparison between bepirovirsen and the pooled placebo group were performed in an exploratory manner. Changes from baseline to day 29 or week 31 for plasma HBV DNA, serum $\mathrm{HBsAg}$ and serum $\mathrm{HBeAg}$ concentrations were logarithmic transformed with base 10. The LOCF method was used to impute missing values. Comparison between bepirovirsen and a pooled placebo group was performed separately for each dose level using an analysis of covariance model with baseline as a covariate and treatment group as a factor. The proportion of participants with reduction in plasma HBV DNA, serum HBsAg and serum HBeAg concentrations of at least 0.5 , 1.0, 1.5 and $2.0 \log _{10}$ at day 29 and week 31 were conducted; comparison between bepirovirsen and placebo was performed using Fisher's exact test. All statistical tests were conducted using two-sided tests with 5\% type I error rates unless otherwise stated. Summaries of HBV RNA and HBcrAg were conducted post hoc.

Reporting Summary. Further information on research design is available in the Nature Research Reporting Summary linked to this article.

\section{Data availability}

Within 6 months of this publication, anonymized individual participant data, the annotated case report form, protocol, reporting and analysis plan, dataset specifications, raw dataset, analysis-ready dataset and clinical study report will be available for research proposals approved by an independent review committee. Proposals should be submitted to www.clinicalstudydatarequest.com. A data access agreement will be required.

\section{References}

24. Crooke, S. T. et al. Integrated safety assessment of 2'-O-methoxyethyl chimeric antisense oligonucleotides in nonhuman primates and healthy human volunteers. Mol. Ther. J. Am. Soc. Gene Ther. 24, 1771-1782 (2016).

25. Levin, A. A., Rosie, Z. Y. \& Geary R. S. in Antisense Drug Technology (ed. Crooke, S. T.) 201-234 (CRC Press, 2007).

26. Yuen, M.-F. et al. Phase $2 \mathrm{a}$, randomized, double-blind, placebo-controlled study of an antisense inhibitor (ISIS 505358) in treatment-naive chronic hepatitis $\mathrm{B}(\mathrm{CHB})$ patients: safety and antiviral efficacy. Hepatology 70, 437-438 (2019).

27. You, S. et al. Short Term Therapy with GSK3228836 in chronic hepatitis B (CHB) patients results in reductions in HBcrAg and HBV RNA: Phase 2a, randomized, double-blind, placebo controlled study. Presentation LP41. AASLD. Presentation LP41 (2020).

28. Dickens, T. et al. Does the galnac liver targeting of antisense oligonucleotides deliver improved clinical efficacy in patients with chronic hepatitis B: A cross study comparison. Frontiers in Drug Development for Viral Hepatitis. Oral abstract O15 (2019).
29. Yuen, M.-F. et al. Hepatitis B virus surface antigen reduction with ISIS 505358 in treatment-naïve chronic hepatitis B patients: a phase 2a, randomized, double-blind, placebo-controlled study. Hepatol. Int. 14, S20-S21 (2020)

30. Yuen, M.-F. et al. Hepatitis B virus (HBV) surface antigen (HBsAg) inhibition with GSK3228836 (ISIS 505358) in chronic hepatitis B (CHB) patients on stable nucleos(t)ide analogue (NA) regimen and in NA -naive $\mathrm{CHB}$ patients: phase 2a, randomized, double-blind, placebo-controlled study. J. Hepatol. 73, S19-S57 (2020).

\section{Acknowledgements}

We thank the patients and study staff without whom this study would not have been possible. Study NCT02981602, currently sponsored by GlaxoSmithKline (study ID 205695), was at the time of the trial, supported by GlaxoSmithKline and sponsored by Ionis Pharmaceuticals, Inc. (ISIS 505358-CS3). Ionis Pharmaceuticals, Inc. were involved in the conceptualization, design, data collection, analysis, decision to publish, and preparation of the manuscript; GlaxoSmithKline were involved in the analysis, decision to publish, and preparation of the manuscript. Study results were previously presented in part at the 70th and 71st Annual Meeting of the American Association for the Study of Liver Diseases ${ }^{26,27}$, HEP DART 2019 (ref. ${ }^{28}$ ), the 29th Conference of the Asian Pacific Association for the Study of the Liver ${ }^{29}$ and the European Association for the Study of the Liver-Digital International Liver Congress ${ }^{30}$. Medical writing and editorial support (in the form of writing assistance, including development of the initial draft based on author direction, assembling tables and figures, collating authors' comments, grammatical editing and referencing) was provided by G. Wallace of Fishawack Indicia Ltd, UK, funded by GSK

\section{Author contributions}

C.F.B. and T.J.K. contributed to the conception or design of the study. M.-F.Y., J.H., J.-W.J., J.-H.Y., Y.-O.K. and S.-J.P. were study investigators and contributed to data acquisition. M.-F.Y., C.F.B., T.J.K., Y. Tami, S.Y., P.Y., Y. Tao, J.C., F.C., R.E., M.P. and D.T. contributed to data analysis or interpretation. All authors revised the manuscript critically for important intellectual content and approved the final version for publication.

\section{Competing interests}

M.-F.Y. has acted as a consultant for AbbVie, Arbutus Biopharma, Assembly Biosciences, Bristol-Myers Squibb, Clear B Therapeutics, Dicerna Pharmaceuticals, GlaxoSmithKline, Gilead Sciences, Janssen, Merck Sharp and Dohme, Springbank Pharmaceutical and Roche, and received grant/research support from Assembly Biosciences, Arrowhead Pharmaceuticals, Bristol-Myers Squibb, Fujirebio Incorporation, Gilead Sciences, Merck Sharp and Dohme, Springbank Pharmaceuticals, Sysmex Corporation and Roche. J.H. received grants/research support from Roche, Yuhan and Gilead. Y.-O.K. and S.-J.P. have nothing to disclose. J.-W.J. has worked as a local consultant for AbbVie, Bristol-Myers Squibb and Gilead Sciences, and received grants/ research support from Yuhan, Hanmi and Sysmex Corporation. J.-H.Y. received grants from AstraZeneca, Bayer, Daewoong and Bukwang. Y. Tami, C.F.B. and T.J.K. are employees and stock/shareholders of Ionis Pharmaceuticals Inc. S.Y., P.Y., J.C., F.C., R.E., M.P. and D.T. are employees and stock/shareholders of GlaxoSmithKline. Y. Tao is an employee of GlaxoSmithKline.

\section{Additional information}

Supplementary information The online version contains supplementary material available at https://doi.org/10.1038/s41591-021-01513-4.

Correspondence and requests for materials should be addressed to Man-Fung Yuen.

Peer review information Nature Medicine thanks the anonymous reviewers for their contribution to the peer review of this work. Alison Farrell was the primary editor on this article and managed its editorial process and peer review in collaboration with the rest of the editorial team.

Reprints and permissions information is available at www.nature.com/reprints. 


\section{Reporting Summary}

Nature Research wishes to improve the reproducibility of the work that we publish. This form provides structure for consistency and transparency in reporting. For further information on Nature Research policies, see our Editorial Policies and the Editorial Policy Checklist.

Please do not complete any field with "not applicable" or n/a. Refer to the help text for what text to use if an item is not relevant to your study. For final submission: please carefully check your responses for accuracy; you will not be able to make changes later.

\section{Statistics}

For all statistical analyses, confirm that the following items are present in the figure legend, table legend, main text, or Methods section. n/a Confirmed

$\bigotimes$ The exact sample size $(n)$ for each experimental group/condition, given as a discrete number and unit of measurement

$\bigotimes$ A statement on whether measurements were taken from distinct samples or whether the same sample was measured repeatedly

$\triangle$ The statistical test(s) used AND whether they are one- or two-sided

Only common tests should be described solely by name; describe more complex techniques in the Methods section.

$\searrow$ A description of all covariates tested

\ A description of any assumptions or corrections, such as tests of normality and adjustment for multiple comparisons

$\square$ A full description of the statistical parameters including central tendency (e.g. means) or other basic estimates (e.g. regression coefficient)

AND variation (e.g. standard deviation) or associated estimates of uncertainty (e.g. confidence intervals)

For null hypothesis testing, the test statistic (e.g. $F, t, r$ ) with confidence intervals, effect sizes, degrees of freedom and $P$ value noted

Give $P$ values as exact values whenever suitable.

Х $\square$ For Bayesian analysis, information on the choice of priors and Markov chain Monte Carlo settings

Х $\square$ For hierarchical and complex designs, identification of the appropriate level for tests and full reporting of outcomes Estimates of effect sizes (e.g. Cohen's $d$, Pearson's r), indicating how they were calculated

Our web collection on statistics for biologists contains articles on many of the points above.

\section{Software and code}

\section{Policy information about availability of computer code}

Data collection No open source computer code was used for data collection

Data analysis SAS version 9.4 was used for data analyses in this study

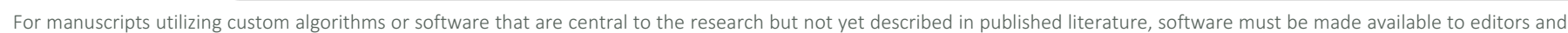

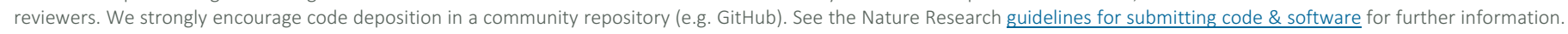

\section{Data}

Policy information about availability of data

All manuscripts must include a data availability statement. This statement should provide the following information, where applicable:

- Accession codes, unique identifiers, or web links for publicly available datasets

- A list of figures that have associated raw data

- A description of any restrictions on data availability

Within 6 months of this publication, anonymized individual participant data, the annotated case report form, protocol, reporting and analysis plan, data set specifications, raw dataset, analysis-ready dataset, and clinical study report will be available for research proposals approved by an independent review committee.

Proposals should be submitted to www.clinicalstudydatarequest.com. A data access agreement will be required. 


\section{Field-specific reporting}

Please select the one below that is the best fit for your research. If you are not sure, read the appropriate sections before making your selection. \Life sciences Behavioural \& social sciences Ecological, evolutionary \& environmental sciences

\section{Life sciences study design}

All studies must disclose on these points even when the disclosure is negative.

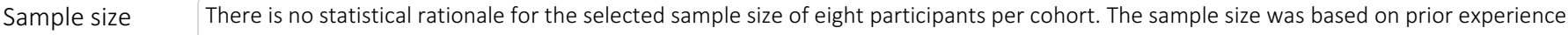
with other members of the drug class to ensure an adequate initial assessment of the safety and tolerability of while minimizing the number of patients unnecessarily exposed to the drug. The protocol included the ability to repeat a cohort if additional data at a specified cohort was warranted.

Data exclusions Figure 2: one patient in the GSK3228836 mg arm discontinued on study Day 8 and is not shown. This patient discontinued treatment and also withdrew from the study; as such they were not assessed beyond Day 8 and are not shown in this figure (exclusion not prespecified). This patient was also excluded from the per protocol population (according to prespecified criteria.

Replication Clinical trial data were derived from a total of 31 patients, and data from all 31 patients who were included in all analyses presented in this manuscript (except for 1 patient described in "Data exclusions" above). It was not feasible to replicate the data as this would require repeating the clinical trial.

Randomization Patients were randomized (3:1 within each dose cohort) to GSK3228836 or placebo according to the randomization schedule (permuted block). The investigator (or designee) obtained the unique study treatment number via an interactive voice/internet response system.

Blinding

All participants, study monitors, study center personnel, and contract research organization personnel were blinded to treatment assignment.

\section{Reporting for specific materials, systems and methods}

We require information from authors about some types of materials, experimental systems and methods used in many studies. Here, indicate whether each material, system or method listed is relevant to your study. If you are not sure if a list item applies to your research, read the appropriate section before selecting a response.

Materials \& experimental systems

$\mathrm{n} / \mathrm{a}$ lnvolved in the study n/a Involved in the study

$\square$ \ Antibodies

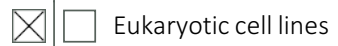

$\triangle \square$ Palaeontology and archaeology

$X \square$ ChIP-seq

$\triangle \square$ Animals and other organisms

$X \square$ Flow cytometry

$\square$ \uman research participants

$\square \bigotimes$ Clinical data

Х $\square$ Dual use research of concern

\section{Antibodies}

Antibodies used

None

Validation

None

\section{Human research participants}

\section{Policy information about studies involving human research participants}

Population characteristics

Adult patients with chronic hepatitis B virus (HBV) infection who fulfilled the following inclusion criteria were eligible to participate in the study: Chronic HBV infection $\geq 6$ months and serum hepatitis B surface antigen (HBsAg) $\geq 50 \mathrm{IU} / \mathrm{mL}$; both hepatitis e antigen ( $\mathrm{HBeAg})$ positive and negative patients could participate. Treatment-naïve patients: Plasma HBV DNA 22x103 IU/mL. On-nucleo(s)tide (On-NA) patients: HBV DNA adequately suppressed (plasma or serum HBV DNA below lower limit of quantification [20 IU/mL]) and currently taking stable tenofovir disoproxil fumarate or entecavir for $\geq 12$ months and expected to continue taking without change through to the end of their participation in this study.

Patients meeting the following criteria were excluded: History of liver cirrhosis and/or evidence of cirrhosis, liver failure, liver 
Recruitment

Ethics oversight disease other than hepatitis B, Gilbert's syndrome or history of laboratory results consistent with Gilbert's syndrome, extrahepatic disorders possibly related to HBV immune complexes, excess alcohol consumption; co-infection with hepatitis $\mathrm{C}$ virus, hepatitis D virus or HIV; screening laboratory values of alanine aminotransferase and aspartate aminotransferase $>5 \mathrm{x}$ upper limit of normal. Treatment-naïve patients: current or prior receipt of anti-HBV NA therapy. Patients who have failed prior interferon treatment $>6$ months prior to screening may be evaluated for possible participation in the study. The full study inclusion and exclusion criteria are detailed in the manuscript's supplementary section.

Thirty-one patients with CHB who were either treatment-naïve (Cohorts 1-3, $n=24$ ) or receiving stable NA therapy (Cohort 4, on-NA patients; $n=7$ ) were enrolled in the study. Patient demographics and baseline characteristics were similar between treatment arms. Demographics and baseline clinical characteristics are shown in Table 1 of the manuscript.

Patients were enrolled from one center in Hong Kong and five centers in the Republic of Korea. The first patient was enrolled February 22, 2017; the last patient was enrolled on the 30 April, 2019; the last patient visit was December 18, 2019; and the study was completed on December 19, 2019.

Details of the independent ethics committees/institutional review boards that approved the study are as follows:

Institutional Review Board of the University of Hong Kong/Hospital Authority Hong Kong West Cluster, Queen Mary Hospital 102 Pokfulam Road, Hong Kong;

Seoul National University Hospital Institutional Review Board, 101 Daehak-ro, Jongno-gu, Seoul 03080, Republic of Korea; Kyungpook National Univeristy Institutional Review Board, 130 Dongdeok-ro, Jung-gu, Daegu 41944, Republic of Korea;

The Catholic University of Korea, Seoul St. Mary's Hospital Institutional Review Board 222, Banpo-daero, Seocho-gu, Seoul 06591, Republic of Korea;

Pusan National University Hospital Institutional Review Board, Department of Internal Medicine, 179 Gudeok-ro, Seo-gu, Busan 49241, Republic of Korea;

Korea University Ansan Hospital Institutional Review Board, 123 Jeokgeum-ro, Danwon-gu, Ansan-Si, Gyeonggi-do 15355 Republic of Korea;

Inje University Busan Paik Hospital Institutional Review Board, Bokji-ro 75, Busanjin-gu, Busan 47392, Republic of Korea

\section{Clinical data}

Policy information about clinical studies

All manuscripts should comply with the ICMJE guidelines for publication of clinical research and a completed CONSORT checklist must be included with all submissions.

Clinical trial registration NCT02981602

Study protocol

The full study protocol can be accessed here: https://www.gsk-studyregister.com/en/trial-details/?id=205695

Data collection

Patients were enrolled from one study center in Hong Kong and five in the Republic of Korea. The first patient was enrolled February 22, 2017; last patient visit was December 18, 2019.

Six doses of GSK3228836 or placebo were administered via subcutaneous injection during the 4-week treatment period on study Days 1, 4, 8, 11, 15, and 22; patients were followed for 26 weeks until Day 211. On Day 29, 7 days after the last dose of study drug (GSK3328836 or placebo), the effects of treatment were assessed. The endpoint assessment for antiviral activity was on Day 29. The final study visit was Day 211. The 'End-of-Study' was defined as the last patient, last visit (Dec 18, 2019).

Blood samples for quantitative $\mathrm{HBsAg}, \mathrm{HBeAg}$, and HBV DNA measurement were collected at screening, pre-dose on Days 1,15 , and 29, and anytime on Days 23, 36, 57, 85, 113, and 211. Blood samples for categorical anti-HBs and anti-HBe antibodies measurement were collected at screening, pre-dose on Days 1 and 29, and anytime on Days 57, 113, and 211.

Outcomes

The primary objective was to examine the safety and tolerability of GSK3228836 administration in treatment-naive participants with chronic hepatitis B infection. This was assessed via incidence of adverse events [AEs], and findings from clinical laboratory tests, vital signs and body weight, physical examination, electrocardiogram, and concomitant medication usage.

Secondary objectives were:

- Examine the effects of GSK3228836 administration on plasma HBV DNA concentration (assessed by change from baseline to Day 29 and Week 31)

- Examine the effects of GSK3228836 administration on serum HBsAg concentration (assessed by change from baseline to Day 29 and Week 31, and the proportion of participants with HBsAg loss at Day 29 and at Week 31)

- Examine the effect of GSK3228836 administration on serum HBeAg concentration in patients who were HBeAg-positive at baseline (assessed by change from baseline to Day 29 and Week 31, proportion of participants with HBeAg loss at Day 29 and at Week 31) - Assess plasma pharmacokinetics of GSK3228836 in patients with chronic HBV infection (assessed by plasma concentrations of GSK3228836 using validated bioanalytical methods)

- Describe the safety and tolerability of tenofovir disoproxil fumarate (and entecavir, if administered) therapy following conclusion of GSK3228836 administration (assessed by the incidence of adverse events after Day 29) 
